\title{
Gunite $^{\mathrm{TM}}$ and Associated Tanks Waste Conditioning System: Description and Operational Summary
}

February 2002

Prepared by

J. A. Emison Informatics, Inc.

B. B. Spencer

B. E Lewis

Oak Ridge National Laboratory 


\section{DOCUMENT AVAILABILITY}

Reports produced after January 1, 1996, are generally available free via the U.S. Department of Energy (DOE) Information Bridge.

Web site http://www.osti.gov/bridge

Reports produced before January 1, 1996, may be purchased by members of the public from the following source.

National Technical Information Service

5285 Port Royal Road

Springfield, VA 22161

Telephone 703-605-6000 (1-800-553-6847)

TDD 703-487-4639

Fax 703-605-6900

E-mail omfp@mtos/fedwpr;d/gpv

Web site http://www.ntis.gov/support/ordemowabout.htm

Reports are available to DOE employees, DOE contractors, Energy Technology Data Exchange (ETDE) representatives, and International Nuclear Information System (NIS) representatives from the following source.

Office of Scientific and Technical Information

P.O. Box 62

Oak Ridge, TN 37831

Telephone 865-576-8401

Fax 865-576-5728

E-mail reports@adonis.osti.gov

Web site http://www.osti.gov/contact.html

This report was prepared as an account of work sponsored by an agency of the United States Government. Neither the United States government nor any agency thereof, nor any of their employees, makes any warranty, express or implied, or assumes any legal liability or responsibility for the accuracy, completeness, or usefulness of any information, apparatus, product, or process disclosed, or represents that its use would not infringe privately owned rights. Reference herein to any specific commercial product, process, or service by trade name, trademark, manufacturer, or otherwise, does not necessarily constitute or imply its endorsement, recommendation, or favoring by the United States Government or any agency thereof. The views and opinions of authors expressed herein do not necessarily state or reflect those of the United States Government or any agency thereof. 


\title{
GUNITETM AND ASSOCIATED TANKS WASTE CONDITIONING SYSTEM: DESCRIPTION AND OPERATIONAL SUMMARY
}

\author{
J. A. Emison \\ Informatics, Inc. \\ B. B. Spencer \\ B. E Lewis \\ Oak Ridge National Laboratory
}

February 2002

\author{
Prepared by \\ OAK RIDGE NATIONAL LABORATORY \\ P.O. Box 2008 \\ Oak Ridge, Tennessee 37831-6285 \\ Managed by \\ UT-Battelle, LLC \\ For the U.S. DEPARTMENT OF ENERGY \\ Under contract DE-AC05-00OR22725
}





\section{CONTENTS}

Page

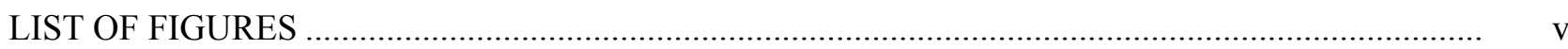

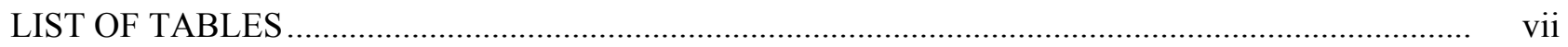

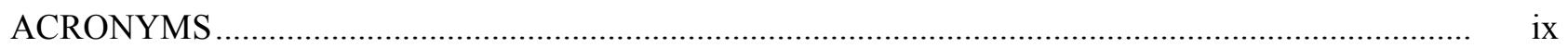

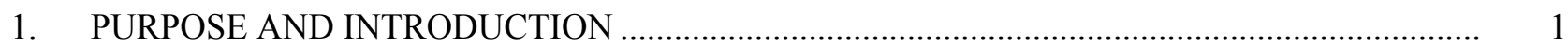

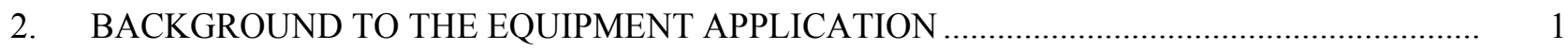

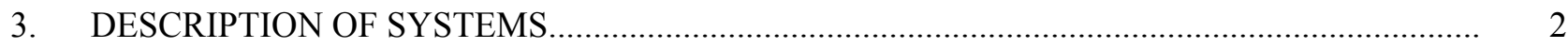

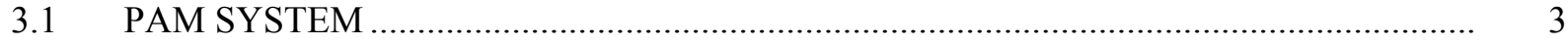

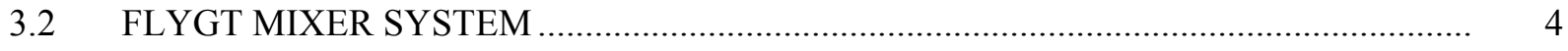

$3.3 \quad$ RUSSIAN PULSATING MIXER PUMP ....................................................................... 5

3.3.1 PMP

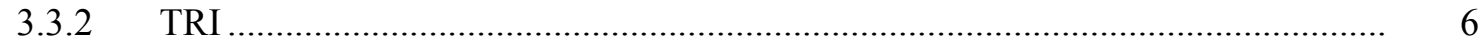

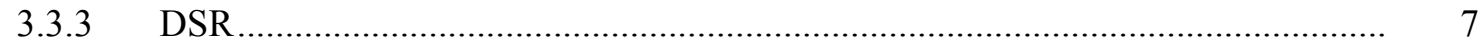

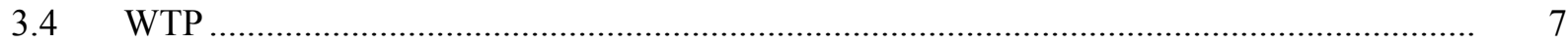

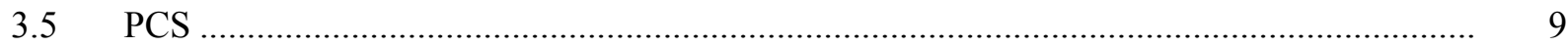

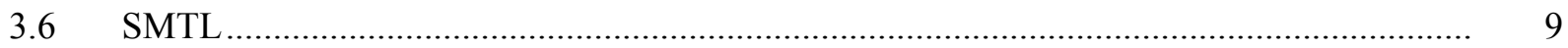

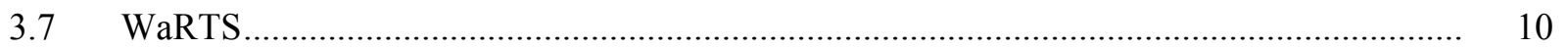

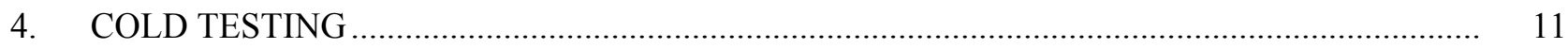

5. OPERATIONAL HISTORY, PROBLEMS, AND MAINTENANCE ....................................... 12

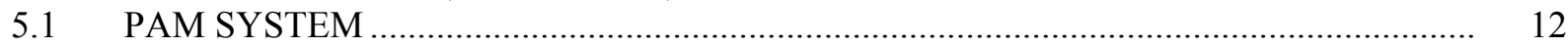

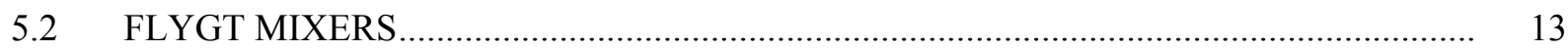

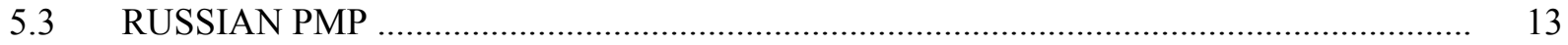

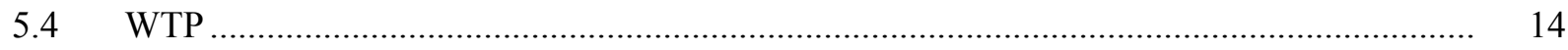

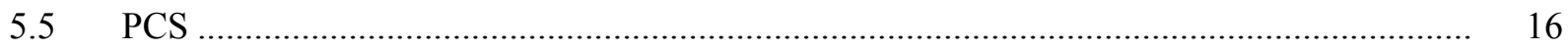

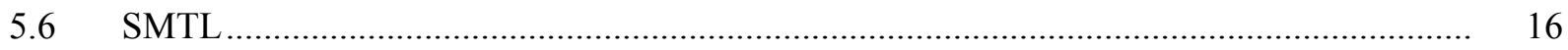

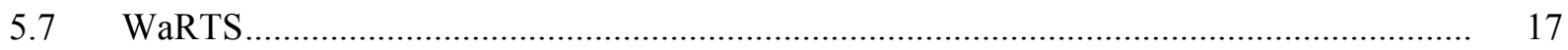

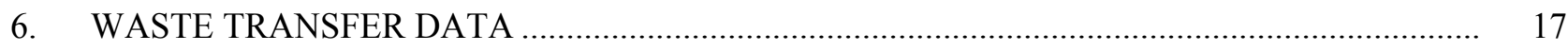

6.1 DISCFLO PUMP TRANSFERS TO MVST FROM W-9 .......................................... 17

6.1.1 Variations in Particle Size Distribution During WTP Operations ........................ 18

6.1.2 Variations in Particle Size Distribution Over One Waste Transfer....................... 23

6.1.3 Effect of Installing the Flygt Mixer on Particle Size Distribution ......................... 25

6.1.4 Settling Data on Sludge Remaining in Tank W-9 at Completion ........................ 26

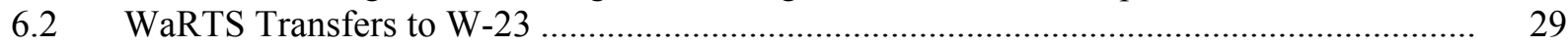

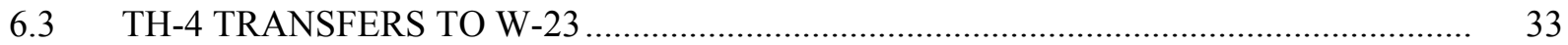

6.3.1 Variation in Particle Size Distribution ................................................................ 33

6.3.2 Settling Data on Tank W-23 Sludge ............................................................... 36 


\section{CONTENTS (continued)}

Page

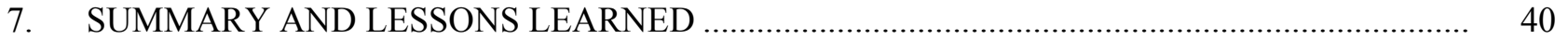

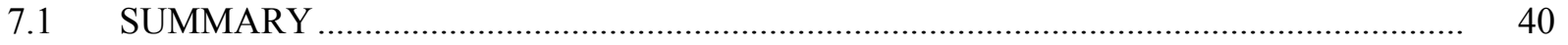

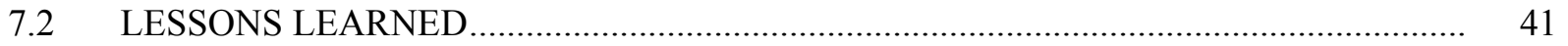

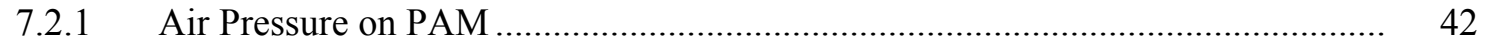

7.2.2 Lightning Arrestor on PAM Controller.......................................................... 42

7.2.3 Adequate Cold Testing of Equipment and Systems........................................... 42

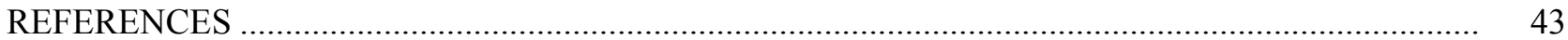




\section{LIST OF FIGURES}

Figure $\quad$ Page

1. General configuration of the waste-conditioning system......................................... 3

2. PAM system before installation in GAAT tank W-9...........................................................

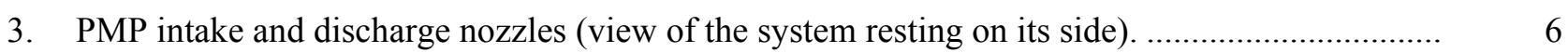

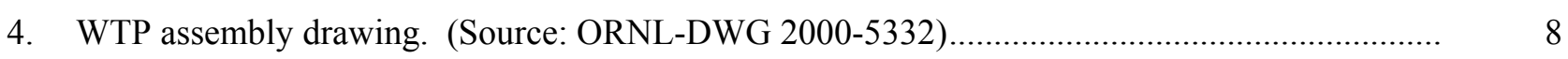

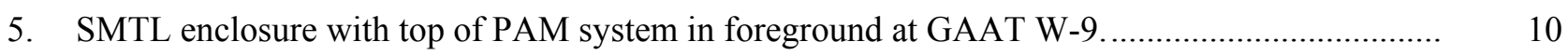

6. WaRTS surge tank and confinement box assembly as it is lifted onto a riser of tank W-9......... 12

7. Distribution of particles measured by the LasenTec instrument during July 22, 1999, slurry transfer from tank W-9 to the MVST. Range of particle size is measured in microns.

8. Number percent distribution based on particle size (LasenTec measurements) for the March 23, 2000, slurry transfer from tank W-9 to the MVST.

9. Weight percent distribution based on particle size (LasenTec measurements) for the March 23, 2000, slurry transfer from tank W-9 to the MVST.

10. Particle size distribution by population for selected WTP transfer operations.

11. Cumulative PSD by population for selected WTP transfer operations.

12. Particle size distribution by weight for selected WTP transfer operations...

13. Cumulative PSD by weight for selected WTP transfer operations.

14. Cumulative PSD by number for the waste transfer of September 9, 1999

15. Cumulative PSD by weight for the waste transfer of September 9, 1999.

16. The effect of the Flygt mixer on cumulative PSD by population.

17. The effect of the Flygt mixer on cumulative PSD by weight.

18. Settling rate of W-9 sludge after completion of MVST transfers, June 28, 2000. Starting sludge volume is about $20 \mathrm{~mL}$.

19. The number of particles measured by the LasenTec instrument during the WaRTS slurry transfer on July 22, 2000 (tank W-9 to ORNL active LLLW tank W-23).

20. PSD by number for selected transfers using WaRTS 


\section{LIST OF FIGURES (CONTINUED)}

Figure

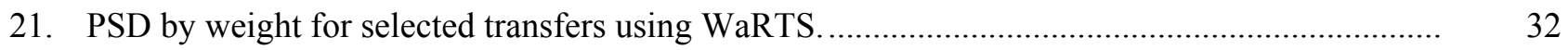

22. Cumulative PSD by weight for selected transfers using WaRTS. ........................................... 32

23. The number of particles measured by the LasenTec instrument during the Russian PMP slurry transfer from tank TH-4 to ORNL active LLLW tank W-23, January 13, 2001.

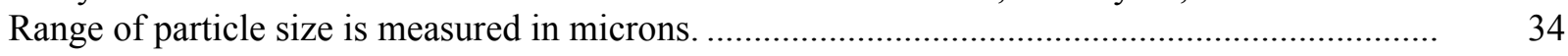

24. Particle-size distribution by population for transfers from TH-4 on January $13,2001 \ldots \ldots \ldots \ldots \ldots . . . . . . . .35$

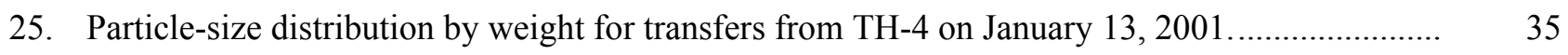

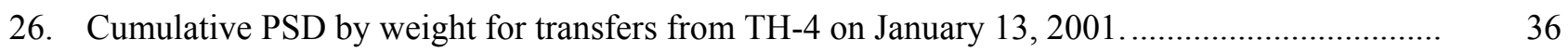

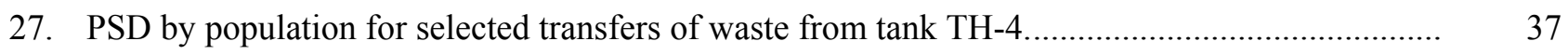

28. PSD by weight for selected transfers of waste from tank TH-4........................................... 37

29. Cumulative PSD by weight for selected transfers of waste from tank TH-4.......................... 38

30. Settling rate on tank W-23 sludge following retrieval of waste from tank TH-4, ca. January 15, 2001. Starting volume is $50 \mathrm{~mL}$ 


\section{LIST OF TABLES}

Table

Page

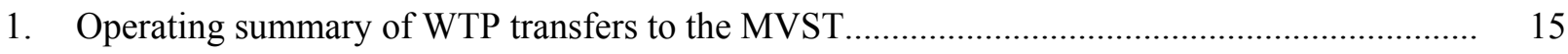

2. Comparison of LasenTec particle size measurements with laboratory results during the W-9 to MVST transfer campaign

3. Beginning and ending dates for waste removal operations ........................................... 21

4. Operating summary of WaRTS transfers to tank W-23 .................................................... 29

5. Run time (in hours) of WCS component systems ........................................................ 40 



\section{ACRONYMS}

ALARA

BVEST

CERCLA

CSEE

DOE

DSR

ECR

EPA

GAAT

HEPA

HWRS

ICPL

LLLW

MVST

NTF

ORNL

PAM

PC

PCS

PMP

PNNL

PSD

PVC

SMTL

SPS

STF

TDEC

TRI

TSS

WAC

WaRTS

WCS

WD\&C

WTP as low as reasonably achievable

Bethel Valley Evaporator Service Tanks

Comprehensive Environmental Response, Compensation, and Liability Act confined sluicing end effector

U.S. Department of Energy

decontamination spray ring

effective cleaning radius

U.S. Environmental Protection Agency

Gunite $^{\mathrm{TM}}$ and Associated Tanks

high efficiency particulate air

heavy waste retrieval system

interconnecting pipeline

liquid low-level waste

Melton Valley Storage Tanks

North Tank Farm

Oak Ridge National Laboratory

PulsAir ${ }^{\mathrm{TM}}$ Mixer

progressive cavity

primary conditioning system

pulsating mixing pump

Pacific Northwest National Laboratory

particle size distribution

polyvinyl chloride

slurry monitoring test loop

supernatant pumping system

South Tank Farm

Tennessee Department of Environment and Conservation

tank riser interface

total suspended solids

waste acceptance criteria

waste retrieval and transfer system

waste-conditioning system

waste dislodging and conveyance

waste transfer pump 


\section{PURPOSE AND INTRODUCTION}

The purpose of this report is to describe and document the function, operational performance, problems encountered, lessons-learned, and overall assessment of the performance of the waste conditioning system (WCS) in the Gunite ${ }^{\mathrm{TM}}$ and Associated Tanks (GAAT) remediation project at the Oak Ridge National Laboratory (ORNL).

The GAAT are located in the main plant area of ORNL in the North and South Tank Farms. These tanks were constructed in 1943 as part of the Manhattan Project during World War II. Each tank in the South Tank Farm (STF) has a 50-ft inside diameter and a capacity of $\sim 170,000$ gal. Each Gunite tank in the North Tank Farm (NTF) has a 25-ft inside diameter with a capacity of $\sim 44,000$ gal. The GAAT were designed to receive radioactive and chemical wastes from ORNL processes. The tanks were constructed of Gunite, which is created by pneumatically spraying concrete over a wire mesh. Following construction, the site was backfilled so the domes of the tanks were covered with $\sim 6 \mathrm{ft}$ of earth. The STF tanks (W-5, $-6,-7,-8,-9$, and -10 ) are set in a $2 \times 3$ array with an east-west axis. The two GAAT in the NTF are on the north side of Central Avenue, and the STF is across the street. One additional Gunite tank, TH-4, is located $\sim 300 \mathrm{ft}$ east of the STF. TH-4 is a smaller, 20 -ft inside diameter tank with a capacity of $\sim 14,000$ gal.

Approximately $90 \%$ of the sludge inventory was removed from the STF tanks during a sluicing campaign in 1982-84 (Autry et al., 1990). Over 95\% of the residual from the original sluicing was removed during the GAAT Remediation Project of 1997-2000. The NTF and STF tanks, as well as tank TH-4 were remediated under the Comprehensive Environmental Response, Compensation, and Liability Act (CERCLA) with regulatory oversight by the U.S. Environmental Protection Agency (EPA) and the Tennessee Department of Environment and Conservation (TDEC).

\section{BACKGROUND TO THE EQUIPMENT APPLICATION}

The GAAT WCS was used to condition the radiochemical sludge slurry and supernatant from nine of the inactive Gunite tanks located in ORNL's main plant area in the Bethel Valley watershed and transfer it to the Melton Valley Storage Tanks (MVST). The sludge was removed from each tank, to the extent practical, with available technologies and consolidated into tank W-9 for cross-site transfer to the MVST. The GAAT sludge will be stored at the MVST and treated for eventual, permanent disposal as part of a separate action along with other ORNL wastes.

The WCS was operated in conjunction with other systems to retrieve slurry from tank W-9 and condition the waste solids for pipeline transfer through the existing ORNL interconnecting pipeline (ICPL) from the STF to the MVST. This pipeline is a 2-in.-diam., buried, double-contained line connecting the main plant waste generating and treatment facilities with the storage tanks. The pipeline is over one mile long and traverses elevation changes of $\sim 200 \mathrm{ft}$.

Due to the critical role the ICPL plays in ORNL's operation, the GAAT slurry transfers had to be completed without plugging or damaging the piping. The WCS was engineered to meet these needs. The WCS consisted of two equipment enclosures, a waste transfer pump (WTP), a mixing system, and the necessary interconnecting piping and valves. The equipment enclosures consisted of a primary conditioning system (PCS) enclosure and a slurry monitoring test loop (SMTL) enclosure. The PCS enclosure houses three samplers, two solids classifiers, pressure sensor and transmitter, and ancillary piping and valves. A sludge grinder was originally planned for the PCS, but subsequent characterization 
data from sludge samples collected during waste transfer operations indicated that the grinder would not be required. The SMTL enclosure contained the necessary in-line slurry monitoring equipment for determination of particle sizes, solids content, viscosity, temperature, and density. The WTP was a submersible pump that could be raised and lowered via a rigid mast.

The WCS was located at STF tank W-9 and was initially operated in conjunction with the PulsAir ${ }^{\text {TM }}$ mixer (PAM) system installed in W-9. The PAMs were used to suspend solids and mix the contents of the consolidation tank. The resulting slurry was transferred through the WCS using the WTP. The WCS was designed to support the transfer of slurry either to the MVST or back to the feed tank. The slurry was initially circulated through the WCS and back into the feed tank until the slurry-monitoring instrumentation in the SMTL enclosure indicated that the slurry contained consistent and acceptable solids content for the transfer to the MVST. The solids classifiers in the PCS were used to ensure that no significant number of solid particles larger than $100 \mu \mathrm{m}$ diam were allowed to enter the Melton Valley transfer line.

The general arrangement of the WCS is illustrated in Fig. 1. The PAMs mixer and instrumentation have been described previously in an ORNL letter report Cold Test Summary Report for Bulk Sludge Retrieval Systems for the Gunite and Associated Tanks (ORNL 1998), and in Comparative Testing of Slurry Monitors (Hylton et al., 1998), and are briefly described in Sect. 3.1. In addition, the results from the hot tests conducted during FY 1999 with the PAM and SMTL have been described in Testing of In-Line Slurry Monitors and Pulsair Mixers with Radioactive Slurries (Hylton and Bayne, 1999).

The WCS was operated in a batch mode, with dilute slurry maintained in suspension by the PAMs while the transfer pump circulated flow through the PCS, SMTL, and a return pipe to tank W-9. Solids classifiers in the PCS removed solids that exceeded a diameter of $100 \mu \mathrm{m}$, in compliance with ICPL criteria, and the oversize particles were automatically backflushed to tank W-9. The in-line slurrymonitoring instrumentation was used to monitor particle size and solids concentration in the slurry downstream of the PCS in the SMTL. After these parameters appeared to be stable and within the criteria for ICPL transfer, valves were positioned to divert flow from the W-9 return pipe into the ICPL, and a batch transfer of up to 40,000 gal was made. Upon completion of a batch transfer, the piping was flushed with clean process water introduced at the PCS. The general configuration of the WCS is displayed in Fig. 1. Each component system of the WCS is further described in Sect. 3.

\section{DESCRIPTION OF SYSTEMS}

The WCS was composed of in-tank mixing systems, pumps, classifiers (filters), samplers, and in-line instrumentation to measure various characteristics of the waste slurry. The mixing systems included the PAM mixer system (W-9), Flygt ${ }^{\mathrm{TM}}$ mixers (W-5 and W-9), and the Russian pulsating mixer pump (TH-4). The Discflo ${ }^{\mathrm{TM}}$ pump was the primary pump used to transfer the slurry through the ICPL to the MVST. A diaphragm pump was used when slurry was transferred a much shorter distance, either from TH-4 to the STF or from tank W-9 to tank W-23, which is across the street and slightly up-slope ( $\sim 5 \mathrm{ft})$ from the STF. A diaphragm pump was installed in the PCS during the final cleanout of tank W-9 and was part of the waste retrieval and transfer system (WaRTS). The PCS unit consisted of particle size classifiers and a sampler set in a steel containment building. The SMTL contained instrumentation to provide real-time monitoring of the suspended solids concentration, density (including dissolved solids), viscosity, temperature, and particle-size range in the waste slurry. Water was added to the waste tanks to clean the tank walls, decontaminate equipment, break up the sludge in the tanks, and as a transport medium. All four sources of water reduced the concentration of the slurry. 


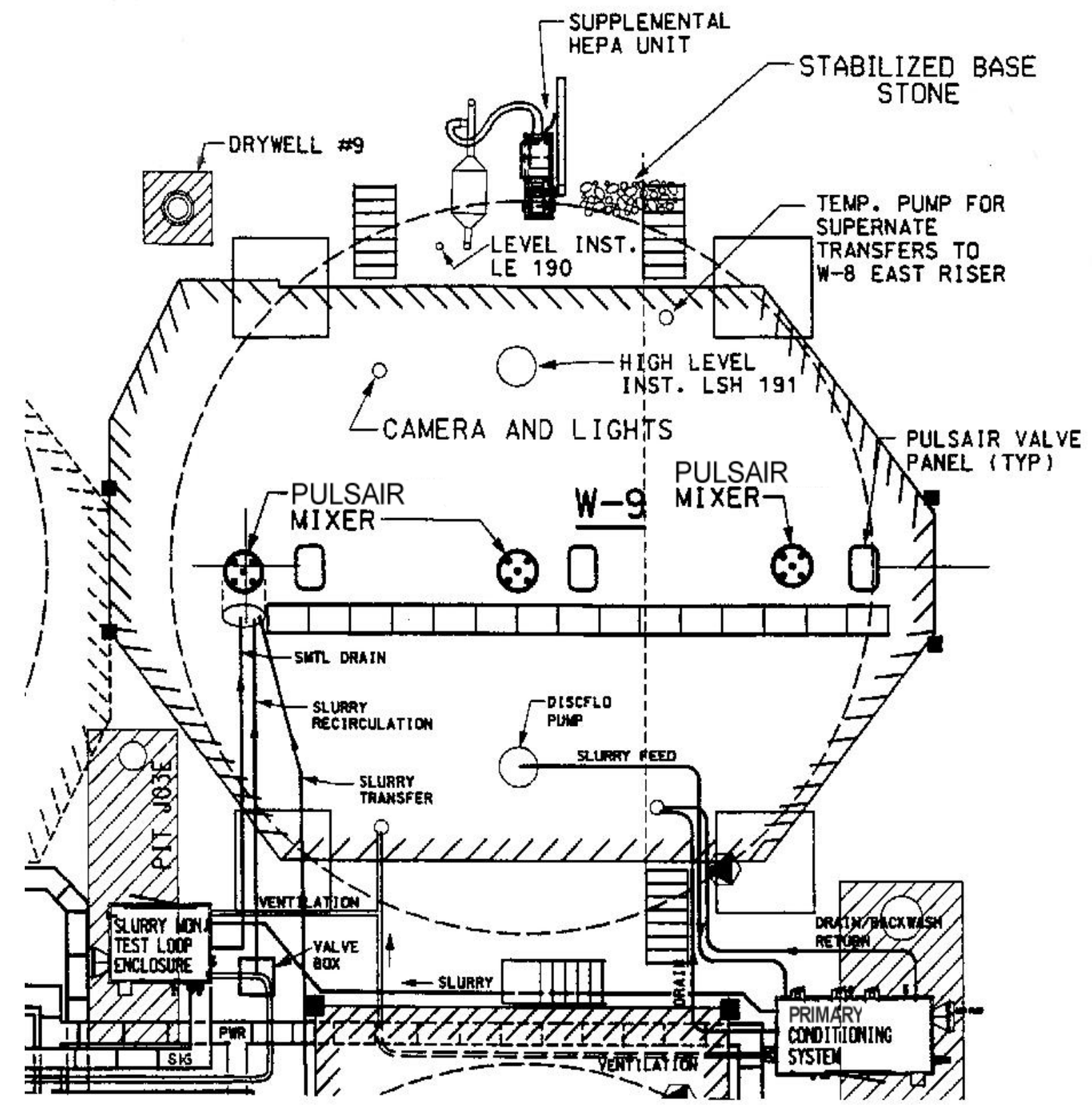

Fig. 1. General configuration of the waste-conditioning system.

\subsection{PAM SYSTEM}

The PAM mixer system comprised three in-tank mixing assemblies, a controller, and necessary tank interface hardware. PulsAir System, Inc., manufactures and markets the PAM system. Each mixing assembly consists of a central accumulator plate with three or four satellite accumulator plates located at the ends of folding arms. The arms provide structural support and also function to convey air from the air-source to the accumulator plates. Each accumulator plate for the ORNL PulsAir mixers consisted of two circular, parallel metal plates spaced $\sim 0.25$ in apart. 
Each accumulator plate was deployed at a predetermined location along the bottom of the tank. The PAM uses timed pulses of compressed air to mix and suspend sludges and sediments in the supernatant. During operation, a pulse of compressed air is discharged through the accumulator plates, causing a large air bubble to form above the plate. Mixing is achieved as liquid is drawn into the low-pressure area under the bubble while liquid above is forced up and away from the rising air bubble. As the bubble breaks at the surface, horizontal forces move the liquid to the tank walls. The liquid travels along the tank walls to the bottom of the tank to complete the mixing cycle before the next pulse occurs.

The PAM system was installed and placed in service at GAAT tank W-9 in June 1998 after a period of cold testing. A view of one of the assemblies prior to its installation into the GAAT is shown in Fig. 2.

ORNL-PHOTO 5235-2000

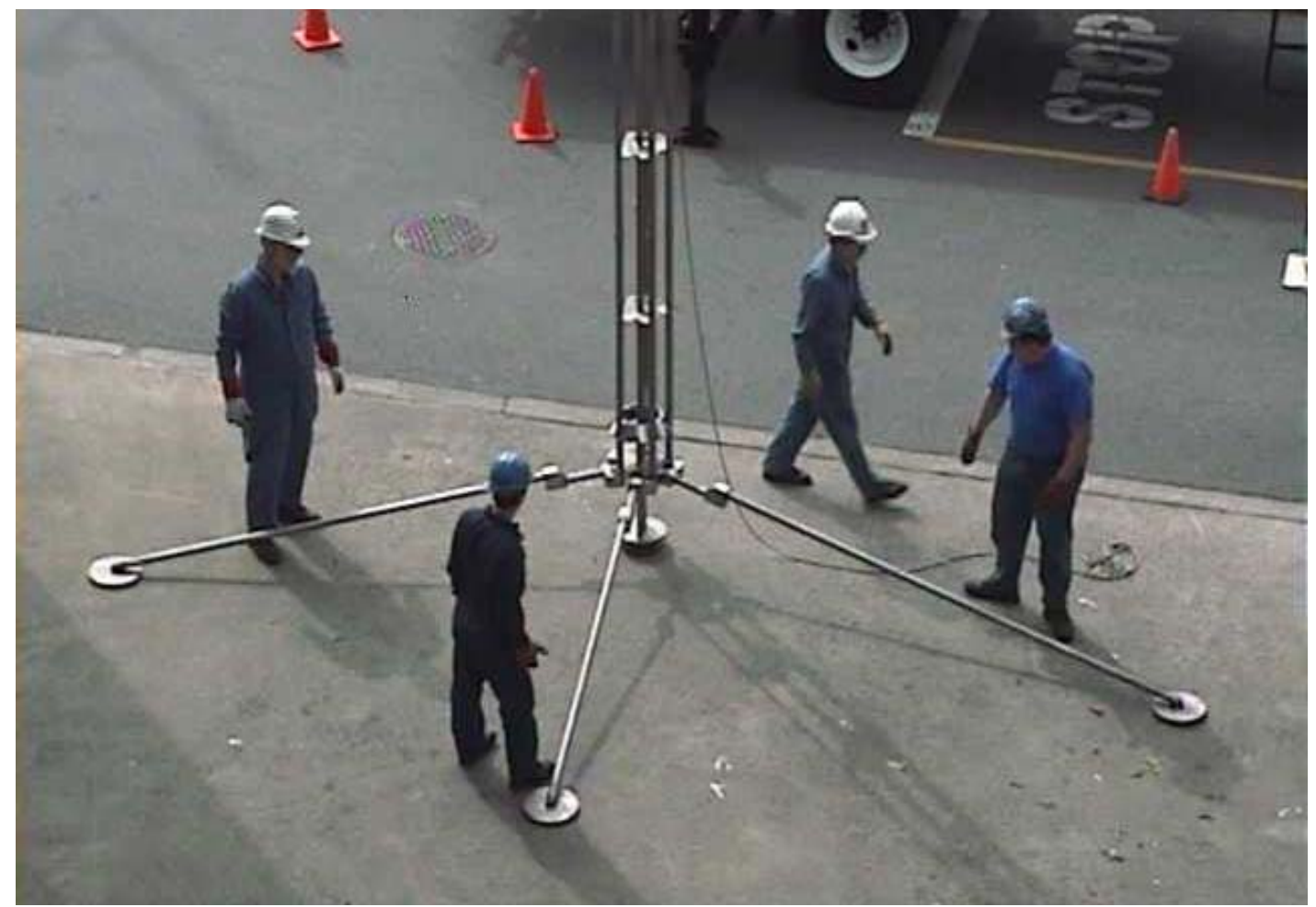

Fig. 2. PAM system before installation in GAAT tank W-9.

\subsection{FLYGT MIXER SYSTEM}

The Flygt mixer system is a Model 4660, axial flow, mechanical (propeller) mixer located at the end of a 25 -ft vertical mast. The mixer and mast are deployed through a tank riser and are supported by a steel platform above the riser. Two mixers were deployed in tank W-5, and one of the two mixers in W-5 was subsequently moved to tank W-9. The mixers were used to agitate the tank contents and suspend sludge in the existing supernatant. The mixers were adjustable in both the horizontal and vertical axes in order to produce the greatest amount of mixing. In its application in the GAAT, the mixers were positioned 
vertically above the sludge level. The mixers were used to impart a velocity to the fluid, which caused solids to be mobilized and suspended. As sludge was removed from tank W-9, reducing the sludge level, the mixer would be periodically lowered. At no time was the mixer operated in the sludge or at the bottom of the tank.

\subsection{RUSSIAN PULSATING MIXER PUMP}

The primary function of the Russian pulsating mixer pump (PMP) was to mobilize and mix solids in tanks containing settled solids and supernatant. The secondary function of the PMP was to keep the solids in suspension while the waste is pumped from the tank. The effectiveness of the mixer is dependent on the size of the tank to be mixed and the characteristics of the waste. Prior to deployment in tank TH-4, the PMP had been tested with simulants thought to bound the key characteristics of actual wastes.

The PMP deployed at ORNL is an adaptation of an existing Russian design in use for radiochemical waste applications in Russia. However, the ORNL PMP was designed to accommodate the unique constraints and requirements of the ORNL GAAT. The PMP system at ORNL consists of three major subsystems: the PMP assembly and control system, the tank riser interface (TRI), and the decontamination spray ring (DSR) (Hatchell et al., 2001).

\subsubsection{PMP}

The PMP comprises a pump chamber, check valve, working-gas supply pipe, discharge manifold, and four discharge nozzles. The pump uses two distinct cycles, fill and discharge, to perform its mixing action. During the fill cycle, vacuum is applied to the pump chamber by an eductor located inside the waste tank to draw liquid into the pump chamber. When the liquid level inside the chamber reaches a predetermined level, the chamber is pressurized with compressed air to discharge the liquid through the discharge nozzles moving it back into the tank to mobilize sludge and settled solids. The maximum working pressure of the PMP is $1586 \mathrm{kPa}(230 \mathrm{psi})$. A large diameter spherical check valve, located at the pump chamber inlet, controls the direction of flow. The PMP can be rotated, using a pneumatic cylinder, through a $90^{\circ}$ arc in alternating clockwise and counterclockwise directions to sweep the entire bottom of the tank. Figure 3 shows the lower end of the PMP lying on its side with the mixing nozzles (upper tapered pipes) and intake for the PMP (lower perforated cylinder). On the top of the PMP (not shown), various shut-off valves are operated in conjunction with an electromechanical air-distribution valve to direct either compressed air or vacuum to the pump chamber.

Supernatant from the waste tank is drawn into the pump chamber through a coarse screen and check valve assembly connected to the bottom of the pump chamber. If a plug occurs in the inlet screen, wash water can be used to rinse the screen. A level sensor located inside the chamber is used to control the vacuum and discharge cycles, with cycle duration dependent on the time required for filling and discharge. Operating frequency and other parameters can be adjusted to generally accommodate the properties of the liquid being mixed. The entire system is remotely controlled and monitored by a laptop computer using Labview ${ }^{\mathrm{TM}}$ software. 


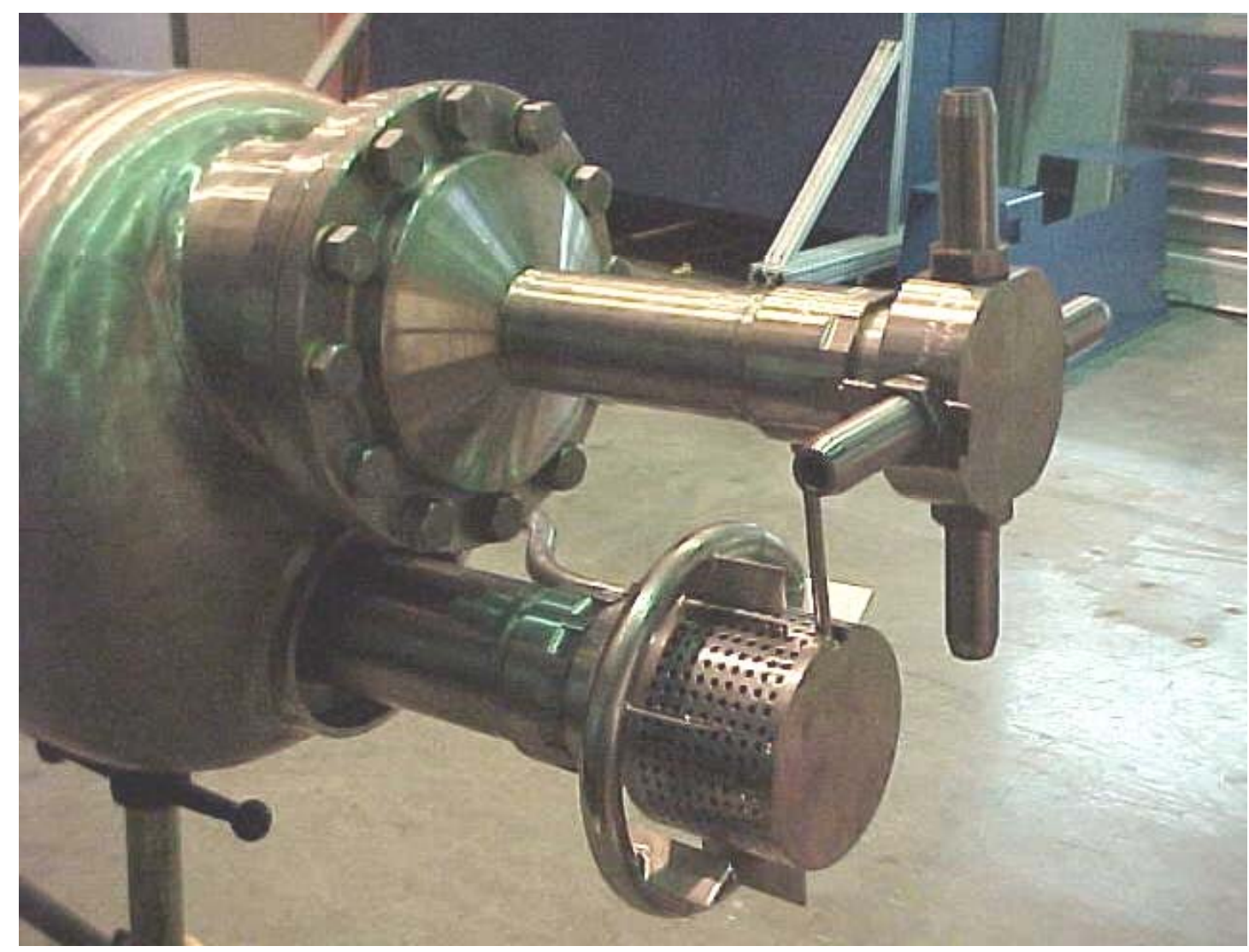

Fig. 3. PMP intake and discharge nozzles (view of the system resting on its side).

\subsubsection{TRI}

The primary functions of the TRI system are to provide (1) an interface between the PMP and the tank riser, (2) the service support platform, (3) an interface between the PMP and site utilities (electrical, air, and water), and (4) housing for the control system. For the deployment in tank TH-4 at ORNL, the TRI frame was connected to a service platform located above the tank. The frame support legs can be used for gross adjustment of the insertion depth of the PMP to accommodate various tank and sludge depths. After installation, the mounting flange of the PMP can be mechanically raised and lowered to further adjust the vertical position of the PMP without breaking containment and to effectively mix fluid at different tank levels. The TRI includes mounting hardware for the control system valves and an enclosure that protects (1) personnel from moving components and (2) the hardware from dust or other environmental debris. The enclosure also permits controlled access to any out-of-tank components requiring routine maintenance. The TRI includes lifting lugs to allow the PMP and TRI assembly to be lifted and inserted into a riser with a single crane. The TRI vertical drive system can be used to adjust the height of the PMP mixing nozzles so that the position of the jets can be varied from $\sim 2.3-58 \mathrm{~cm}$ above the tank floor.

The PMP is connected to the control valves by flexible hoses. Because the PMP oscillates in a rotary motion, the support structure for the control valves was designed to avoid interference with the rotation of 
the PMP, and to limit stress and wear on the hoses and associated connections. The major system components of the TRI include

- $\quad$ PMP frame base plate. The PMP is mounted to a thick, rectangular plate. This part includes a long circular section, which serves as an attachment point for a flexible bellows that interfaces with the DSR attached to the tank riser.

- Mechanical lifting drive. The PMP frame base plate is attached to four screw-type Duff Norton $^{\mathrm{TM}}$ mechanical actuators to allow the PMP depth to be varied over a range of $\sim 56 \mathrm{~cm}$. The mechanically coupled actuators are driven through rotating shafts and mitre gear boxes with a $1 / 2$ HP Duff Norton motor.

- PMP support frame. A modular frame is the primary support structure for the PMP, drive system, mounting plate, control valves, and electrical boxes.

\subsubsection{DSR}

The function of the DSR system is to remove surface contamination from the in-tank components of the PMP during withdrawal of equipment from the tank. Process water or other commonly used decontamination fluids may be used for system decontamination. The DSR was designed to mount directly to the tank riser using a mounting flange; the DSR includes mounting holes for the spray nozzles and hoist rings. Six $65^{\circ}$ fan-type spray nozzles are mounted around the circumference of the DSR to allow the PMP in-tank components to be washed during equipment extraction. The jets provide complete coverage of the equipment. The jet nozzle assemblies include a nozzle holder and flow straightener. A manual control valve connected to the inlet of the manifold can be closed when the spray ring is not in use to maintain tank containment. In addition, a check-valve downstream of the manual valve protects against inadvertent contamination caused by backflow while the manual valve is open. The manifold can be connected to a high-pressure pump or lower-pressure supply with proper choice of nozzle size. All components are rated to at least $13,800 \mathrm{kPa}(2,000 \mathrm{psi})$. Internal decontamination of the PMP was accomplished by the fresh water flushing-system.

\subsection{WTP}

The WTP was required to deliver a flow rate of $\sim 50-60$ gallons per minute (gpm) over a diverse range of conditions. During recirculation, discharge pressure is limited to a few pounds per square inch necessary to overcome resistance in the PCS classifier and the remaining WCS recirculation loop piping and instrumentation. During a waste transfer, the pump must maintain a constant flow rate at a discharge pressure of $>200$ psi. However, the discharge pressure must be limited to 300 psi to avoid overpressurizing the ICPL. To maintain radiation exposures as low as reasonably achievable (ALARA) and promote contamination control, the pump must require little or no maintenance and accommodate provisions for secondary confinement as well as for shielding personnel from radiation.

Progressive cavity (PC) pumps are the baseline technology implemented by ORNL for this type of operation. PC pump limitations in this application are (1) the high cost of equipment, (2) relatively large size of the pumps, (3) high cost and length of time required for siting and constructing double-contained pump pits, (4) potentially high maintenance cost and effort due to wear when pumping abrasive solid materials, and (5) special considerations for over-pressure protection.

The WTP selected for use in the WCS is a submersible, disk pump manufactured by Discflo ${ }^{\mathrm{TM}}$ Corporation of El Cajon, California. The Discflo pump combines the relatively simple design of a centrifugal pump with the capabilities of a PC pump to satisfy the WTP requirements. The Discflo pump 
uses a series of rotating disks in place of a typical impeller. The disks operate on a viscous drag-boundary layer principle. Advantages in this application are the ability to pump abrasive solids and entrained air with little or no internal wear to the pump, the ability to handle large solid particles, and little or no increase in the discharge pressure even when the output line is blocked. The WTP is equipped with a variable-frequency drive to control pump speed and thus the desired discharge flow.

For the GAAT application, the Discflo pump and 125-hp submersible electric motor are supported from a steel deployment mast mounted on a structural platform above the tank. The mast was adjustable to accommodate varying slurry depths in the tank. It included a confinement boundary to provide radioactive contamination control and to maintain negative pressure inside the tank. A sketch of the pump and mast assembly is shown in Fig. 4. The pump and motor assembly can be deployed through a 30 -in-diam riser and is adjustable over a range of $\sim 20-26 \mathrm{ft}$ below the riser in 1-ft increments.

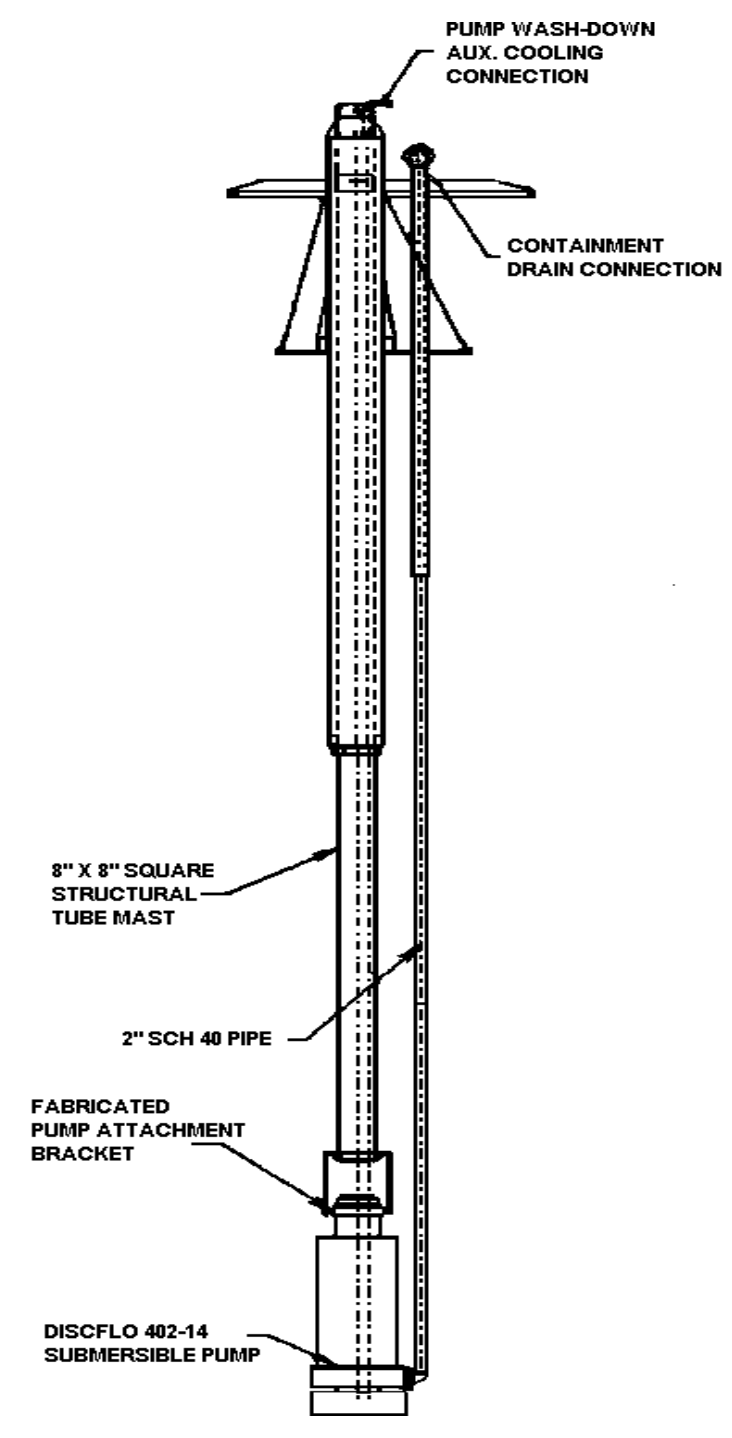

Fig. 4. WTP assembly drawing. (Source: ORNL-DWG 2000-5332) 


\subsection{PCS}

The PCS consists of a confinement enclosure that houses classifiers, samplers, a pressure transmitter, a process water flush connection, and associated valves and piping. The enclosure has a HEPA-filtered air inlet and a ventilation connection with a back draft damper that maintains a negative pressure by connection to the off-gas system of tank W-9. The PCS also uses a sump to collect any leaks that may occur from the process piping and drain them back to the tank. A wash-down capability is installed to remove gross contamination inside the enclosure in the event of a catastrophic leak from the primary piping. A sludge grinder was originally planned for the PCS, but was omitted based on sludge characterization data that showed the solids retrieved from the GAAT were primarily $<100-\mu \mathrm{m}$-diam particles, which met the acceptance criteria for transfer through the ICPL.

The PCS that receives the stream from the WTP was designed to filter out large solid particles. The two classifiers, manufactured by Orival, Inc., were installed in parallel so either could serve as a backup if one was off-line. During operation, the classifiers can operate automatically to flush large solids back to tank W-9 using either an operator-selected time interval or a pre-determined pressure drop. Isolock ${ }^{\mathrm{TM}}$ in-line samplers, manufactured by Bristol Equipment Co., were located both upstream and downstream of the classifiers. The filters were used only briefly as explained in Sect. 5.5. A spool piece was installed downstream of the classifiers to accommodate future installation of a grinder or other equipment. An additional Isolock sampler was installed downstream of the spool piece location. Valving installed in the PCS permitted the classifiers to be bypassed if desired.

Double-contained piping was installed from the WTP discharge to the confinement enclosure and from the PCS discharge to the SMTL enclosure. Steel pipe was used for secondary containment for aboveground piping in the WTP loop (with steel primary piping). PVC pipe was used as secondary containment for some under-ground transfer piping applications (with steel primary piping). The classifier back-flush drain was also doubly contained to minimize the potential for a release. System instrumentation and controls permitted remote monitoring and operation of the samplers and valves from the GAAT operations control trailer.

\subsection{SMTL}

Criteria for transfer of waste slurries through the ICPL require the concentration of suspended solids to be $<5 \mathrm{wt} \%$ and the maximum particle size to be $100 \mu \mathrm{m}$ (Hylton and Bayne, 1999). The SMTL was designed to provide real-time monitoring of the radioactive slurry conditions before and during waste transfers to the MVST. Instrumentation monitored the slurry particle size, density, suspended solids concentration, viscosity, temperature, and flow. These monitoring capabilities were designed to operate remotely to minimize radiation dose to workers.

The test loop was designed for operation with pressures up to $300 \mathrm{psig}$, which was consistent with the maximum operating pressure of the Discflo pump and the ICPL. The system was designed so that any or all of the slurry monitoring instruments could be used or bypassed as operating conditions required. The test loop was housed in a steel enclosure, which served as secondary containment. The system also contained an Isolock $^{\mathrm{TM}}$ sampler. Figure 5 shows the SMTL module in the midground and the air injection hoses attached to one of the three PAM assemblies, which is installed in a riser of tank W-9. 


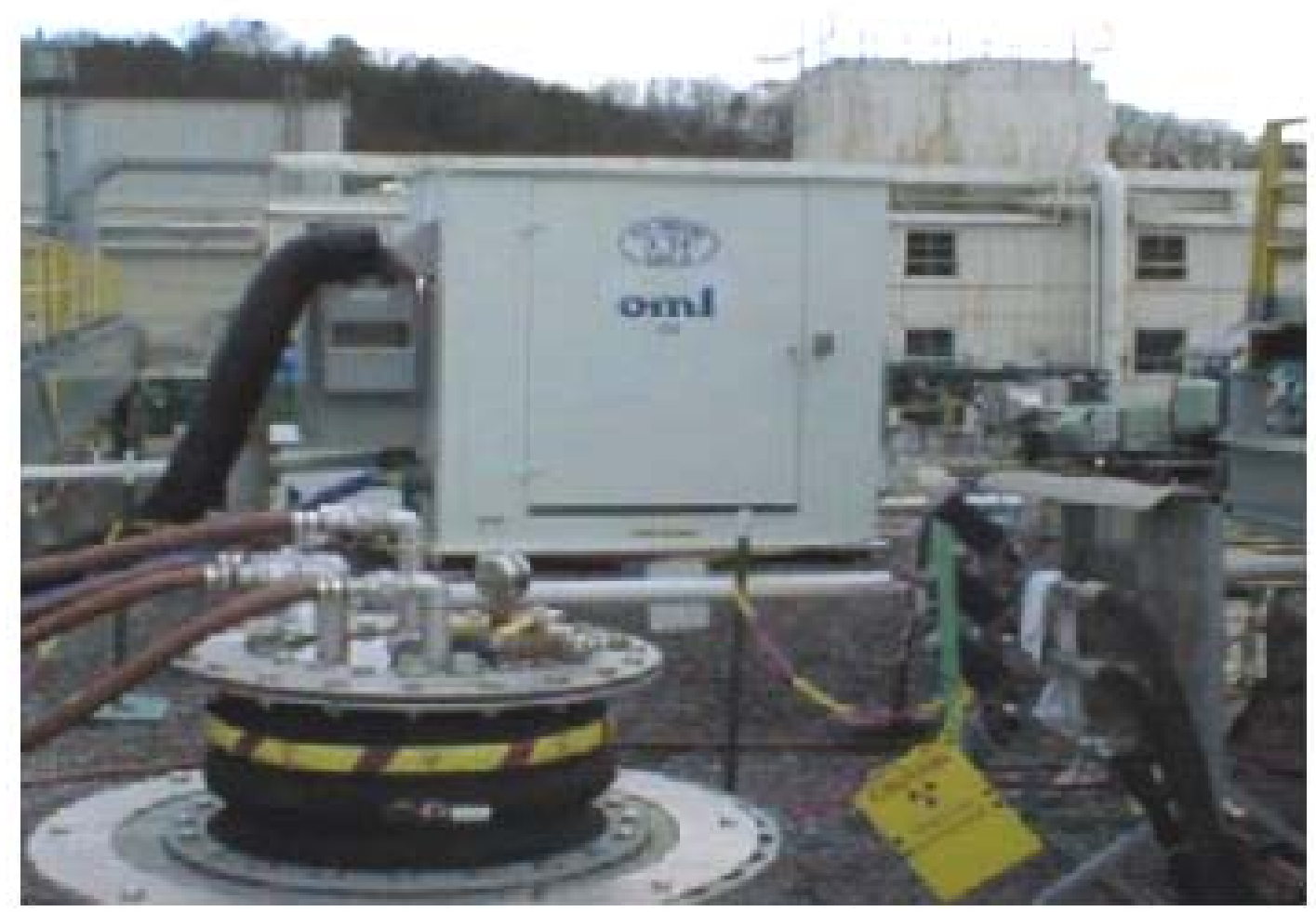

Fig. 5. SMTL enclosure with top of PAM system in foreground at GAAT W-9.

\subsection{WaRTS}

The WaRTS was part of the heavy waste retrieval system (HWRS) that was used to remove the sludge remaining in tank W-9 after the light sludge had been removed. It was installed on June 22, 2000, after the PAMs, Flygt mixers, and WTP were removed from the tank. The WaRTS included the supernatant pumping system (SPS) to control the flow of supernatant to the waste holding tank and for transfers of slurry (Innovative Technology 2000).

The WaRTS included a small surge tank that sat on or within a riser on tank W-9. It interfaced with the waste dislodging and conveyance (WD\&C) system to move material from tank W-9 into the holding tank for subsequent transfer to the active system via a positive displacement diaphragm pump installed in the PCS enclosure. WaRTS received the waste slurry output of the WD\&C jet pump operating in tank W-9. Operators managed the waste level in the WaRTS surge tank to ensure a steady supply and flow to the under-ground pipeline to tank W-23. Operators also ensured that larger particles, that were initially mobilized by the jet pump and subsequently settled out in the surge tank, were returned to tank W-9. They also adjusted the water content as necessary to achieve the proper concentration of solids.

The discharge of the WaRTS was fed to the PCS. The system was designed with fittings to receive recycled supernatant from either tank W-8 or an auxiliary holding tank and to receive waste from tank TH-4 through an above-ground doubly contained pipeline. "Recycled" supernatant was added to the WaRTS surge tank to smooth the flow and to reduce the concentration of solids as required. 
The secondary containment system for portions of the WaRTS was constructed of 0.25 -in carbon steel and was positioned on the $\mathrm{W}-9$ work platform above a 34-in-diam riser to which it was sealed. The secondary containment system provided containment for the surge tank and the associated piping and valving. Any leakage drained back into the tank.

Three remote controlled, air operated, solenoid-activated valves and a check valve were housed in the WaRTS secondary containment system. These valves controlled the flow of supernatant and could be positioned such that the supernatant was recirculated to the supernatant supply tank, added to the waste stream, or added directly to the holding tank. Check valves were included to prevent any slurry from entering the supernatant supply. Provisions for a clean process water flush of the system and decontamination of the containment system were also made.

Figure 6 shows the WaRTS surge tank and confinement box assembly as it was installed on tank W-9. The surge tank was designed for installation on an existing 24 -in-diam riser. It provided a working volume of $\sim 200$ gal, a settling volume of $\sim 75$ gal, and $\sim 50$ gal of capacity above the working high level. The tank assembly features are

- a remotely controlled drain valve in the bottom of the tank which was designed to fail open, connected with a 4-in-diam drain line that empties back into the source tank,

- level instruments that provided process information and signals used by either the control system to automatically control the process or the operator to manually control the process,

- a camera system with a remotely controllable variable light source, and a remote capability to wash and rinse the camera lens to remove any material splashed during the pumping and waste transfer process,

- a remotely operable air and water sparger in the bottom of the tank, and

- a 4-in-diam overflow or vent which prevented over-filling or pressurization of the tank.

\section{COLD TESTING}

Two of the WCS component subsystems, the WaRTS and the PMP, were subjected to extensive cold testing prior to deployment in the STF and TH-4. The SMTL was extensively tested with clean process water and surrogate waste prior to installation at the STF. Testing of the PAMs, Flygt mixers, and the Discflo pump (waste transfer pump) was relatively limited, although vendor testing of the Discflo pump was witnessed by ORNL personnel and cold checkout of the PAM system was done to test the deployment and retraction features of the system. In addition, the PAM system was tested at Pacific Northwest National Laboratory (PNNL) during development, before delivery of the equipment to ORNL. 


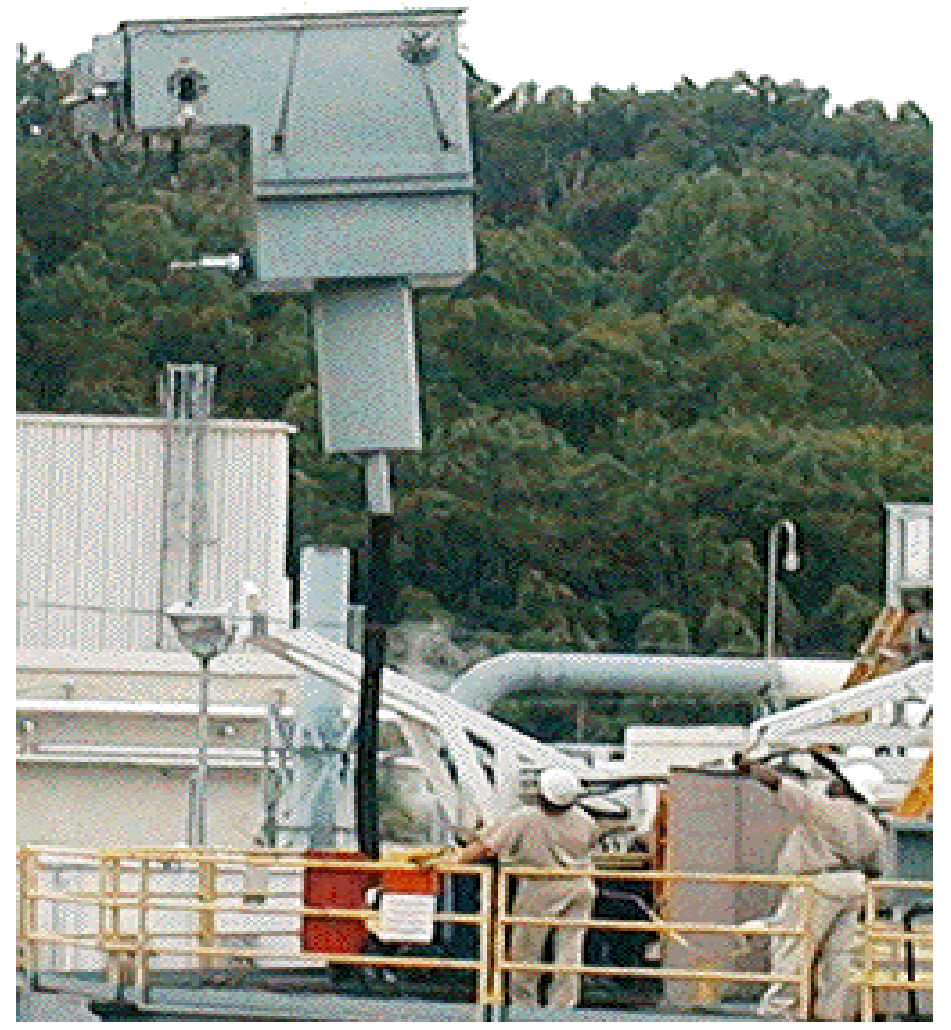

Fig. 6. WaRTS surge tank and confinement box assembly as it is lifted onto a riser of tank W-9.

\section{OPERATIONAL HISTORY, PROBLEMS, AND MAINTENANCE}

\subsection{PAM SYSTEM}

The PAM system operated for more hours with less maintenance or interruption caused by other factors than any other system used during the remediation of the GAAT. The system was installed in tank W-9 in June 1998. It was first used for remedial operations in December 1998 and continued in service through the entire MVST waste transfer campaign to the end of March 2000. The system logged a total of $\sim 2,390 \mathrm{~h}$ of operations.

Only one maintenance action was required on the PAM system. One of the air supply pipes became clogged with sludge during installation. At that time, the sludge was probably $>2 \mathrm{ft}$ deep and would eventually accumulate to a depth of $>4 \mathrm{ft}$ as sludge was transferred into W-9. The system was forced down into the sludge and was not operated for several months between the initial testing phase in June and regular operation beginning in December. It was thought that sludge hardened in the air supply pipe to one of the accumulator plates and blocked the air supply. The air supply line to the plugged accumulator plate was temporarily refitted for a process water supply, and the line was cleared using hydrostatic pressure. 
Lightning was also suspected of disrupting the PAM system by causing the control system to stop functioning. This situation was observed several times during inclement weather. It was never determined precisely how the system was disrupted, perhaps by power surges. After each outage, the controls were reset and the system restarted.

\subsection{FLYGT MIXERS}

The Flygt mixers were not part of the original design of the WCS. Originally, the two mixers were installed in tank W-5 as the only sludge mobilization system needed for that tank. During installation, the high-chrome hardened steel propeller on one unit was damaged after coming into contact with the tank dome as the mixer was being lowered into the tank. The combined weight of the mixer and mast assembly broke one blade from the propeller, but caused no visible damage to the motor shaft or seals. Following replacement with a stainless steel propeller, the two mixers were reinstalled in tank W-5.

Project managers became convinced of the need to augment the PAM system during the final cleanout of tank W-9. Both functionality and availability of the Flygt mixers led to their use with the WCS. The PAM system tended to mobilize sludge to produce inverted cone-shaped zones of influence in the area of each accumulator. The Flygt mixer was positioned to direct the flow of sludge across the accumulators of the PAM system, thus creating a synergistic effect in regard to sludge suspension.

One Flygt mixer was moved from tank W-5 to tank W-9 in August 1999 and was operated a total of $\sim 322.5 \mathrm{~h}$ from September 1999 to the end of March 2000. The system was reliable, requiring little or no maintenance. However, operators of the system considered the Flygt mixers to be somewhat temperamental in regard to fault trips. Typically, fault trips were caused by an overcurrent to the motor. The system could be reset and restarted fairly quickly at slightly less current and consequently a lower operating speed. The Flygt mixers used in the GAAT were also considered slightly underpowered for this application, with motors rated at only $15 \mathrm{hp}$. More powerful off-the-shelf models that could be deployed through the existing risers were not available at the time of the GAAT remediation project.

\subsection{RUSSIAN PMP}

When the Russian PMP was delivered to ORNL it was inspected and tested at the ORNL Tank Technology Cold Test Facility. During inspection, certain issues arose that required resolution prior to testing the system (Innovative Technology 2000; Lewis and Randolph 2001). These issues included the following:

- repair of welds on the pressure vessels for PMPs 2 and 3 caused by undetected weld defects;

- cleanup of weld slag on PMP 1 level sensor thimble;

- replacement of broken and loose components on the TRI and PMP 1;

- installation of grease fittings on deployment cradle pivot points; and

- rewiring of TRI drive motor brake.

Tests were conducted to assess the functionality of the TRI, DSR, control system components, transport cradle, support fixtures, and contamination control features. All hardware components performed satisfactorily after making the following minor modifications and replacement of select components to improve operation and reliability.

- Relays were added to control modules. 
- Emergency stops were added.

- Limit switches on the TRI were replaced with waterproof switches.

- Control system programming was revised to improve functionality and simplify operation.

- The lifting points for the TRI were moved from the top of the frame to the TRI base plate so that the combined PMP and TRI could be lifted as a single unit.

Performance testing revealed that the effective cleaning radius (ECR) of the system was $\sim 7 \mathrm{ft}$ when operating with a 90 psig air supply and while holding stationary. When using oscillating jets on various types of sludge surrogates, the ECR ranged from $\sim 5.5$ to $8 \mathrm{ft}$. The nozzle size did not dramatically affect the ECR over the range of materials tested.

The Russian PMP system operated the shortest duration of all the components used in conjunction with the WCS. Tank TH-4 was the smallest of all the Gunite tanks with a capacity of $\sim 14,000$ gal. The TH- 4 cleanout campaign lasted just three days and the PMP was operational for $\sim 25 \mathrm{~h}$. The system removed $\sim 82 \%$ of the sludge from the tank. Removal of the remaining sludge was not required to satisfy tank risk criteria.

\subsection{WTP}

After presenting technical problems at the startup of operations in tank W-9, the WTP (Discflo pump) performed reliably. During the initial testing and installation in tank W-9, an electrical breaker on the $480-\mathrm{V}$ power supply to the pump tripped open after a few hours of operation and could not be reset. Troubleshooting revealed a problem with the variable frequency drive, which was sent to a local authorized service contractor for diagnosis. The service contractor determined that the drive was damaged by extremely low line impedance and a current imbalance across the phases on the ORNL power system. Installation of an isolation transformer and line reactor corrected the situation.

The Discflo pump was observed to overheat when liquid levels in the tank were below the elevation of the motor housing. Because the pump was designed to operate submerged like a sump-pump, air-cooling was inadequate. An external pump was used to provide a flow of $\sim 5$ gpm of supernatant over the pump housing for cooling. The liquid was delivered through a wash-down pipe installed as an integral part of the Discflo pump's support mast. Supernatant was used for cooling to avoid the addition of clean water to the tank, which would then require subsequent treatment.

The WTP operated $\sim 180 \mathrm{~h}$ in recirculation and transfer modes. The total amount of waste transferred to the MVST was $\sim 483,300$ gal, of which $\sim 60,500$ gal was wet sludge. Waste transfer operations were intermittent, based on available capacity in the liquid low-level waste (LLLW) system. Table 1 displays the operating summary for the WTP transfers to the MVST. 
Table 1. Operating summary of WTP transfers to the MVST

\begin{tabular}{|c|c|c|c|c|c|c|}
\hline $\begin{array}{l}\text { Transfer } \\
\text { number }\end{array}$ & $\begin{array}{c}\text { Transfer } \\
\text { date }\end{array}$ & $\begin{array}{l}\text { Slurry } \\
\text { density } \\
(\mathrm{g} / \mathrm{mL})\end{array}$ & $\begin{array}{l}\text { Slurry volume } \\
\text { (gal) }\end{array}$ & $\begin{array}{l}\text { Sludge } \\
\text { transferred } \\
\quad(\text { gal })^{l}\end{array}$ & $\begin{array}{l}\text { Total activity } \\
\text { transferred } \\
(\mathrm{Ci})^{2}\end{array}$ & $\begin{array}{l}\text { Particle size } \\
<105 \mu \mathrm{m} \\
\text { (percent) }^{3}\end{array}$ \\
\hline 1 & $5-25-99$ & 1.024 & 10,435 & 753 & 395 & Not available \\
\hline 2 & $6-11-99$ & 1.037 & 25,704 & 2,501 & 3,074 & Not available \\
\hline 3 & $6-22-99$ & 1.044 & 23,795 & 3,381 & 2,845 & 99.953 \\
\hline 4 & $7-22-99$ & 1.044 & 27,220 & 3,868 & 5,703 & 99.955 \\
\hline 5 & $7-28-99$ & 1.058 & 26,144 & 3,521 & 5,210 & 99.976 \\
\hline 6 & $8-17-99$ & 1.050 & 41,860 & 5,316 & 5,562 & 99.945 \\
\hline 7 & 9-09-99 & 1.023 & 37,570 & 5,396 & 6,374 & 99.964 \\
\hline 8 & $9-28-99$ & 1.047 & 29,813 & 3,694 & 2,803 & 99.972 \\
\hline 9 & $10-26-99$ & 1.055 & 25,100 & 4,395 & 3,591 & 99.978 \\
\hline 10 & $11-17-99$ & 1.028 & 25,330 & 3,657 & 2,382 & 99.963 \\
\hline 11 & $12-07-99$ & 1.024 & 36,659 & 4,833 & 2,810 & 99.982 \\
\hline 12 & $1-13-00$ & 1.045 & 23,048 & 3,434 & 2,308 & Not available \\
\hline 13 & $1-20-00$ & 1.042 & 24,300 & 2,520 & 1,813 & Not available \\
\hline 14 & $2-11-00$ & 1.043 & 21,000 & 3,000 & 2,039 & 99.944 \\
\hline 15 & $2-18-00$ & 1.042 & 24,027 & 2,756 & 1,842 & 99.926 \\
\hline 16 & $3-09-00$ & 1.029 & 27,120 & 2,222 & 1,330 & Not available \\
\hline 17 & $3-23-00$ & 1.039 & 25,066 & 2,233 & 1,460 & 99.976 \\
\hline 18 & $3-30-00$ & 1.049 & 29,086 & 2,971 & 2,021 & 99.958 \\
\hline Total & & & 483,277 & 60,451 & 53,562 & \\
\hline
\end{tabular}

${ }^{1}$ Volume of sludge transferred is an estimate of the amount removed from the GAAT. It is based on an initial estimate of 88,000 gal of wet sludge stored in the GAAT. Based on the remedial investigation data, the sludge had an average bulk density of $1.26 \mathrm{~g} / \mathrm{mL}$ and a solids content of $31 \%$ by weight.

${ }^{2}$ Total activity transferred to the MVST is an estimate in Curies. Calculations are based on the total activity in $\mathrm{Bq} / \mathrm{ml} \times 103 \mathrm{~mL} / \mathrm{L} \times 3.875 \mathrm{~L} /$ gal $\times$ volume transferred in gal $\times 2.7 \times 10^{-11} \mathrm{Ci} / \mathrm{Bq}$.

${ }^{3}$ Percentage by number, from LasenTec measurements. 
The Discflo pump was designed to achieve pressures of at least $200 \mathrm{psig}$, which was estimated to be the minimum pressure needed to complete slurry transfers through the ICPL at the design flow rate. However, the actual pressure of the transfers was in the range of 160 to 190 psig.

During early operations in tank W-9, what seemed to be a small crack in the impeller housing or shaft casing between the motor and impeller began to visibly spray water. Later, it was determined that the leak was at the flange between the motor and pump impeller. The leak stopped before completion of the transfer campaign and did not appear to interfere with waste transfer operations.

\subsection{PCS}

The PCS was used primarily to collect slurry samples during waste transfers to the MVST and to tank $\mathrm{W}-23$. The capability to physically reduce particle size was never installed because it was not needed, as previously described. The particle size classifiers (filters) were used only during the initial transfer of material to the MVST and were subsequently bypassed. The filters frequently clogged and reduced the downstream pressure. The pressure drop triggered the automatic backflush cycle. The filters were installed in parallel to allow one to be backflushed while the other continued to operate. The filters clogged almost constantly, making it virtually impossible to maintain the pressure and flow needed for the transfer. Apparently the sticky, cohesive nature of the sludge particles contributed to the blockage of the filters. For these reasons, use of the filters was discontinued. Data from the LasenTec particle size analyzer as well as laboratory analytical data supported the fact that virtually no solids $>100 \mu \mathrm{m}$ were present in the slurry, which led to the conclusion that the classifiers were not required.

During the final cleanout of tank W-9 (WaRTS operation), and during the waste transfers from tank TH-4, the PCS was used as secondary containment for the diaphragm pump used to transfer slurries from W-9 to Bethel Valley Evaporator Service Tanks (BVEST) W-23. The diaphragm pump (discussed in Sect. 3.7) was installed in place of the grinder because the particle size data indicated that the grinder was not needed. This data is summarized in Table 1.

\subsection{SMTL}

The SMTL provided measurements of particle size distribution during all three phases of the slurry transfer campaign in the STF and in tank TH-4. The SMTL was operated continuously while slurry was being moved from either tank W-9 or TH-4 to either destination (the MVST or W-23) in the ORNL active LLLW system. The operating times included $\sim 180 \mathrm{~h}$ during WTP operations; $\sim 88.5 \mathrm{~h}$ during WaRTS operation; and $\sim 30 \mathrm{~h}$ during the Russian PMP operation in tank TH-4.

Project operators and management relied on the SMTL as real-time evidence that the waste slurry met ORNL LLLW system waste acceptance criteria (WAC) with respect to particle size. The LLLW WAC limited the largest particle size to $\sim 100 \mu \mathrm{m}$. This limit was applicable during the inter-valley transfers to minimize the risk of plugging the 2-mile-long pipeline. The section on waste transfer data (Sect. 6.) shows the real-time measurements of particle size and some comparison with laboratory analyses.

The SMTL also provided data on the solids content of the slurry. This was important data because the STF Safety Analysis Report (Holder 1993) established limits on the activity $\left({ }^{90} \mathrm{Sr}\right.$ equivalent) of the slurry that could be pumped through the system, and the activity was related to solids content. While the SMTL provided a useful trend in total solids content (i.e., change in value), the actual percentage of solids was not a reliable measurement (i.e., absolute value). Project operators and managers chose instead to rely on 
analytical laboratory data for solids content. Therefore, laboratory analysis of slurry samples was used to estimate the quantity of solids transferred and the amount of residual material in the tanks following the final batch transfers.

\subsection{WaRTS}

The WaRTS was installed in the east riser of tank W-9 on June 22, 2000, as described in Sect. 3.7. The installation of interconnecting piping, signal wiring, electrical power, etc., was checked out and tested over a period of about 3 weeks. The first supernatant transfer to tank W-23 was accomplished on July 13, 2000. A total of 14 slurry transfers to tank W-23 occurred from the period July 18 to September 7, 2000. The total operating time (supernatant and slurry transfers) for WaRTS was $\sim 88.5 \mathrm{~h}$.

Based on operating logs and a review of project maintenance and configuration control documentation, WaRTS was a reliable system that functioned as designed without maintenance or modification during the final, brief W-9 clean-out campaign. The only operational problem was a minor one where the air sparger in the surge tank was partially plugged and did not provide as vigorous mixing as planned. Operators were able to compensate for the problem, and attempts were not made to modify or repair the sparger.

\section{WASTE TRANSFER DATA}

Three phases of waste transfer from the STF occurred. In the first phase, 18 transfers of waste from tank W-9 to the MVST using the Discflo pump occurred. During the second phase, the WaRTS was used for 14 transfers of waste from tank W-9 to tank W-23. A diaphragm pump provided the motive force during this phase of operation. The third phase consisted of waste transfer using a diaphragm pump in conjunction with the Russian PMP to transfer wastes from tank TH-4 to tank W-23.

\subsection{DISCFLO PUMP TRANSFERS TO MVST FROM W-9}

For reasons previously stated, the most meaningful and reliable data generated by the SMTL was particle size measurements. These measurements were critical to meeting the ORNL LLLW system WAC, which limited particle size in order to avoid plugging the ICPL. The data from the LasenTec instrument were verified in several instances by independent laboratory measurements. Table 2 compares the key measurements of the fraction of particles with diameters $<105 \mu \mathrm{m}$ with laboratory results of total suspended solids (TSS) $>50 \mu \mathrm{m}$ in diameter. The laboratory results were based on a $500 \mathrm{~mL}$ sample accumulated during the course of a 25,000-40,000 gal transfer (i.e., a proportional sample). The LasenTec data represent an average value of all measured particles during the entire transfer event. Both data sets indicate the presence of only a miniscule percentage of solid particles of a size that had the potential to cause problems (i.e., over the $100 \mu \mathrm{m}$ limit set by the WAC).

Table 1, of Sect. 5.4, summarizes information about the number, timing, and volume of material moved during each of the Discflo pump transfers to the MVST. 
Table 2. Comparison of LasenTec particle size measurements with laboratory results during the W-9 to MVST transfer campaign

\begin{tabular}{rccc}
\hline $\begin{array}{c}\text { Transfer } \\
\text { date }\end{array}$ & $\begin{array}{c}\text { Particle size }^{l} \\
<105 \mu \mathrm{m}(\%)\end{array}$ & $\begin{array}{c}\text { Sample identification }^{2} \\
\text { (sample number, request number) }\end{array}$ & $\begin{array}{c}\text { Laboratory result }^{3} \\
\text { (wt \% of TSS }<50 \mu \mathrm{m})\end{array}$ \\
\hline $8 / 17 / 99$ & 99.945 & $990818-011$, IPA9737 & 99.989 \\
$9 / 28 / 99$ & 99.972 & $990929-009$, IPA9828 & 99.984 \\
$10 / 26 / 99$ & 99.978 & $991027-078$, IPA9912 & 99.979 \\
$11 / 17 / 99$ & 99.963 & $991118-011$, IPA9926 & 99.978 \\
\hline
\end{tabular}

${ }^{l}$ Percentage particle size is averaged over the entire transfer batch (25,000 gal to 41,000 gal range) using a LasenTec instrument measurement. Note, this refers to percent by total number of particles.

${ }^{2}$ Samples were analyzed at the ORNL Radioactive Materials Analytical Laboratory.

${ }^{3}$ Laboratory results are based on a proportional $500 \mathrm{~mL}$ sample collected during the batch transfer.

Figure 7 provides an example of the relative quantities of various particle size ranges as measured by the LasenTec in-line particle size analyzer for the July 22, 1999, slurry transfer from tank W-9 to the MVST. This was the fourth of 18 transfers to the MVST. The particle counts were relatively constant for the selected size ranges measured during the transfer. The primary perturbation in the graph reflects that the Discflo pump tripped at 14:40, the fault was cleared and the pump restarted at 14:50.

Particle size distribution is more clearly shown on scatter graphs. Figs. 8 and 9 display particle size distributions by number percent and weight percent, respectively. These graphs show data from the March 23, 2000, slurry transfer from tank W-9 to the MVST. This was the $17^{\text {th }}$ of 18 transfers to the MVST.

\subsubsection{Variations in Particle Size Distribution During WTP Operations}

The particle size distribution data were checked for changes that might have occurred over the course of the waste transfers from W-9 to the MVST. Massive quantities of particle size distribution (PSD) data were recorded by the LasenTec M600P system during waste transfer operations. Each waste slurry transfer from tank W-9 to the MVST took several hours. Measured distributions were recorded at a frequency of one complete distribution per minute. Each distribution comprised 38 characteristic size intervals and a count-rate (counts/s) of particles falling within each size interval. Normalized distributions (i.e., count-rate in a particular interval divided by the sum of the count-rates in all intervals) give a close approximation to a number-frequency (or population) distribution. 


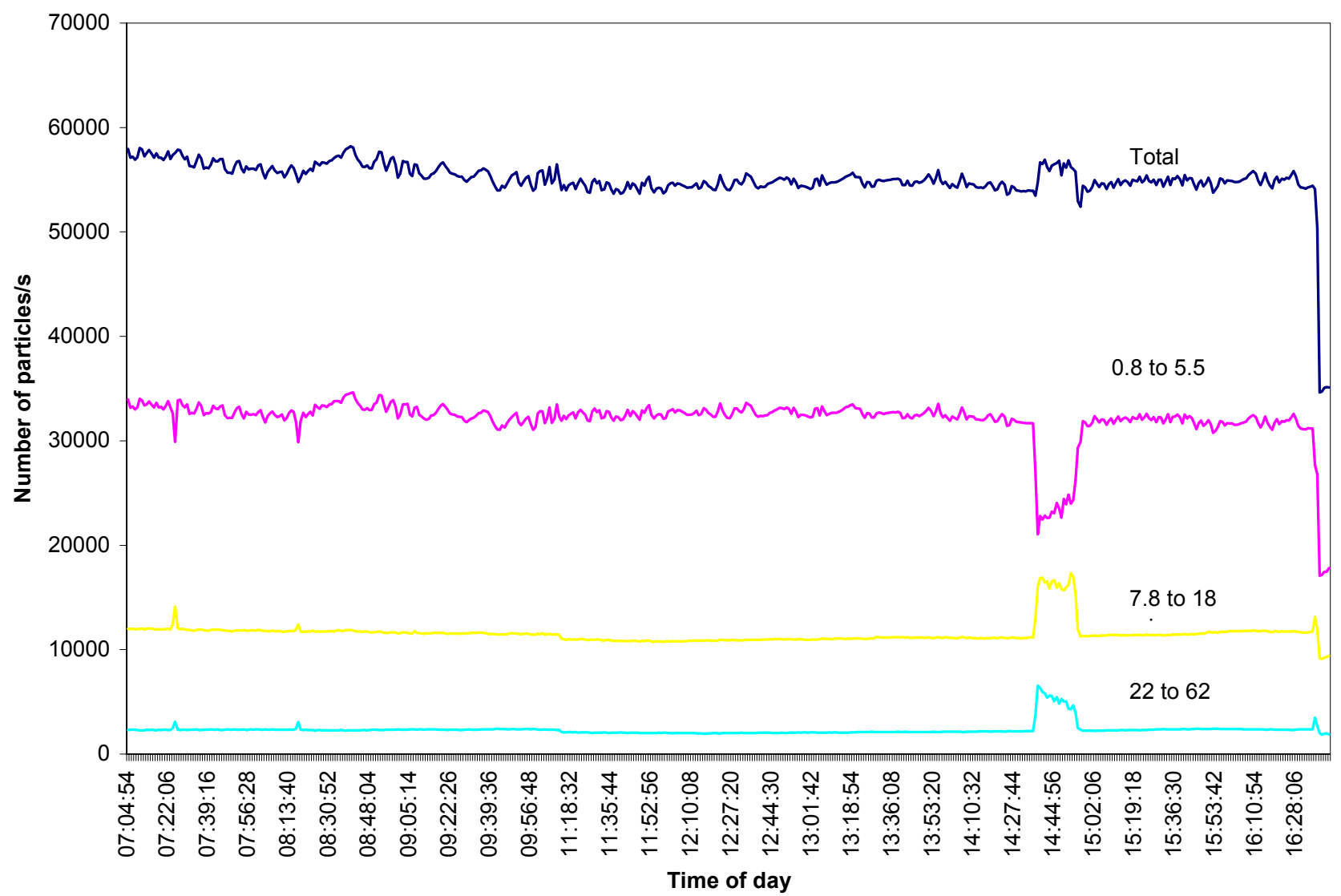

Fig. 7. Distribution of particles measured by the LasenTec instrument during July 22, 1999, slurry transfer from tank W-9 to the MVST. Range of particle size is measured in microns. 


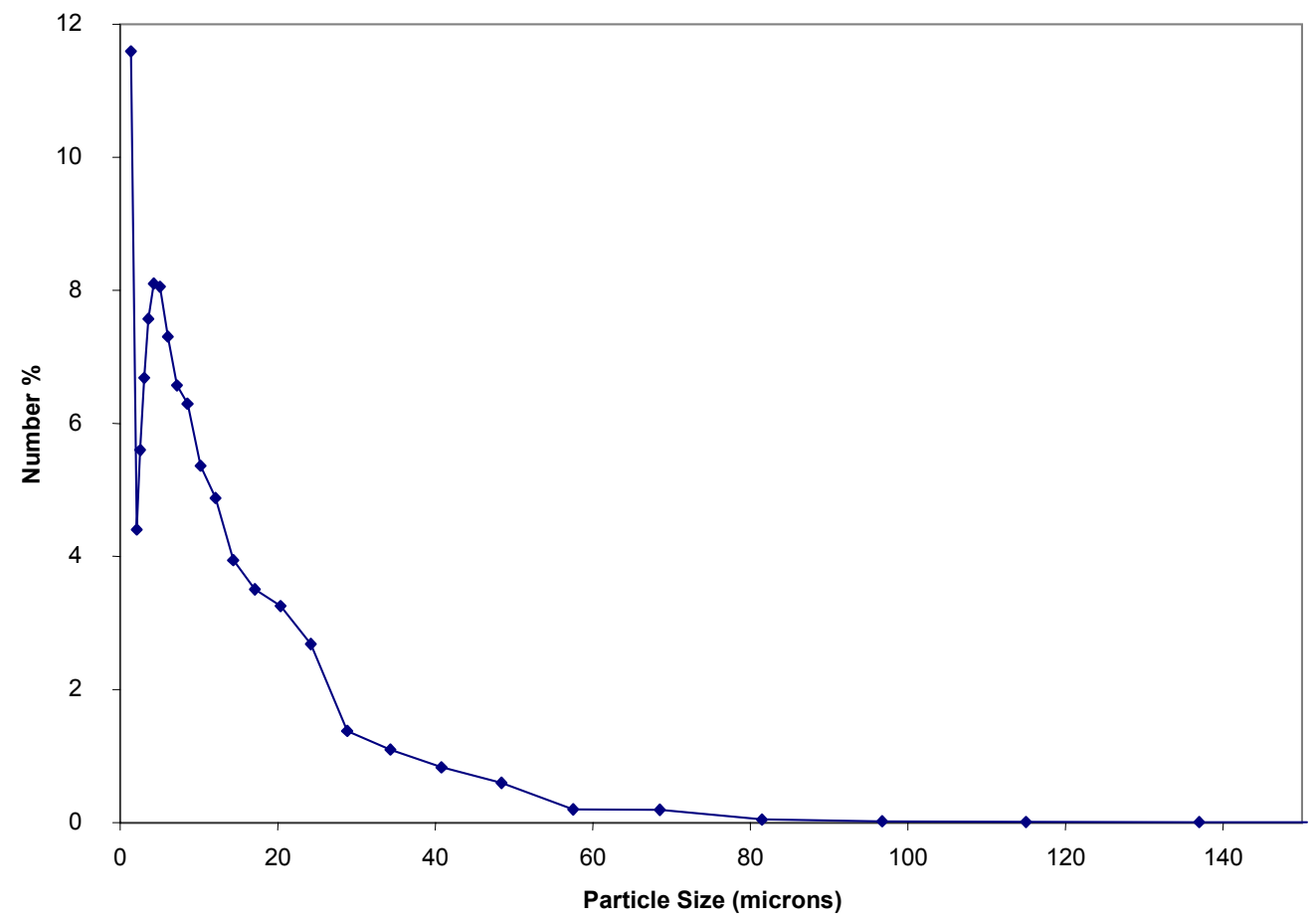

Fig. 8. Number percent distribution based on particle size (LasenTec measurements) for the March 23, 2000, slurry transfer from tank W-9 to the MVST.

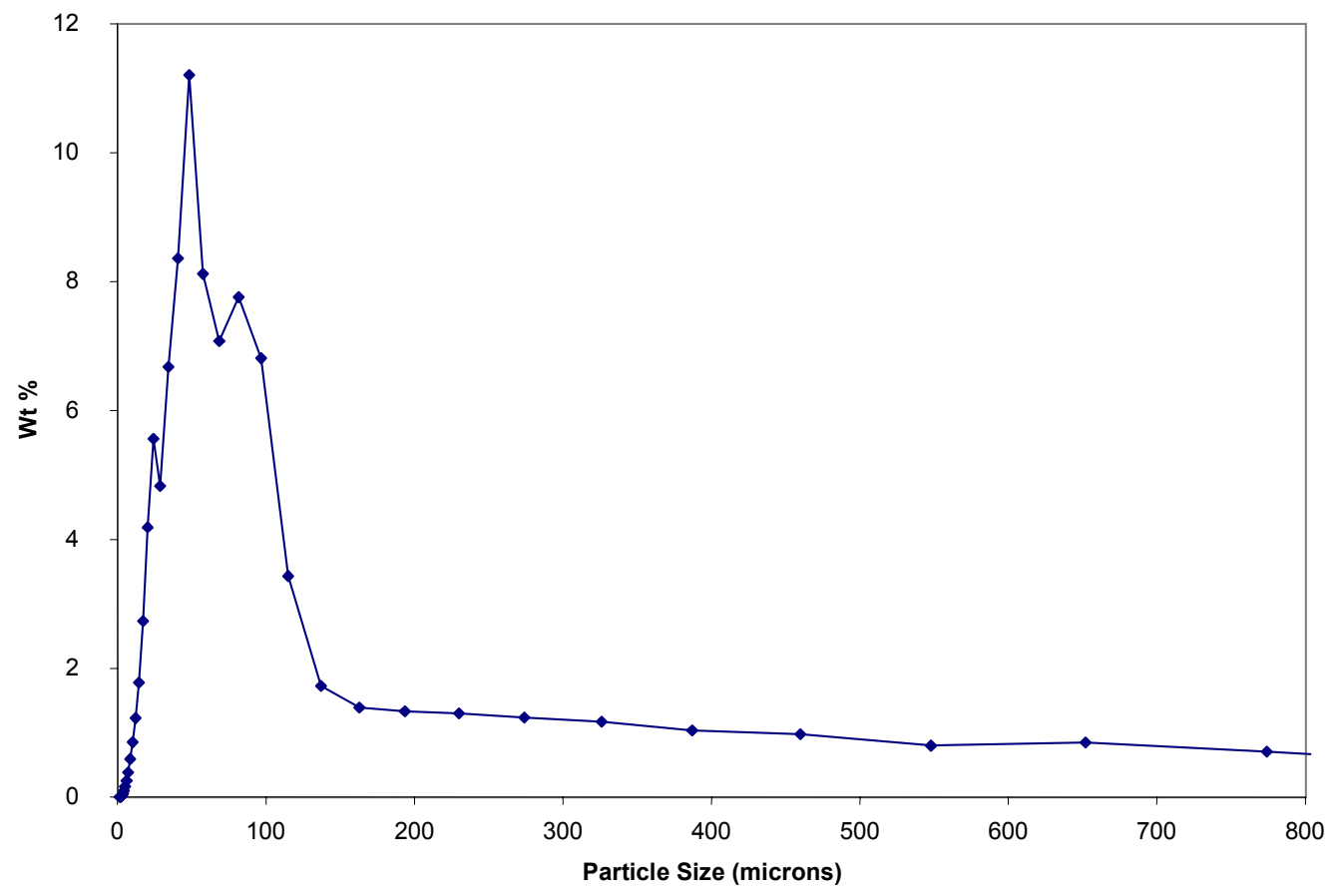

Fig. 9. Weight percent distribution based on particle size (LasenTec measurements) for the March 23, 2000, slurry transfer from tank W-9 to the MVST. 
WTP transfers from W-9 were intermittent, with the first occurring on May 25, 1999, and the last on March 30, 2000 (see Table 1 in Sect. 5.4). Waste had been transferred by sluicing from tanks W-6, W-5, and W-7 over a period ending April 5, 1999. Thus the waste from these tanks was accumulated with the waste in tank W-9 before WTP operations began. After WTP operations began, intermittent sluicing operations transferred wastes from tanks W-8 and W-10 to tank W-9. This resulted in waste being partially removed from tank W-9 and transferred to the MVST, and waste being transferred from W-8 or W-10 to W-9 between the removal transfers. Alternation between removal of waste from, and addition of waste to, W-9 was not a uniform cycle. For reference, the time intervals for waste removal (sluicing) operations are summarized in Table 3.

Table 3. Beginning and ending dates for waste removal operations

\begin{tabular}{lrr}
\hline & \multicolumn{2}{c}{ Waste removal } \\
\cline { 2 - 3 } Tank & \multicolumn{1}{c}{ Begin } & \multicolumn{1}{c}{ End } \\
\hline W-6 & $6-18-98-98$ \\
W-5 & $9-17-98$ & $11-23-98$ \\
W-7 & $10-23-98$ & $4-05-99$ \\
W-10 & $6-04-99$ & $10-26-99$ \\
W-8 & $11-29-99$ & $3-13-00$ \\
W-9 & $7-13-00$ & $9-07-00$ \\
\hline
\end{tabular}

${ }^{1}$ WTP transfers from W-9 to the MVST started 5-25-99 and ended 3-30-00, as presented in Table 1.

${ }^{2}$ WaRTS transfers from W-9 to W-23 started 7-18-00 and ended 9-7-00, as presented in Table 4.

As noted earlier, 18 waste transfers occurred using the WTP that spanned a period of 10 months. As an approach to seeking systematic changes in the particle size distribution of the transferred wastes, the average PSDs of three widely spaced waste transfers were compared. To avoid bias in selecting a particular instantaneous PSD from a given transfer operation which typically took several hours, the timeaveraged PSD for the entire transfer was taken as representative. Figure 10 illustrates the average PSD on three dates that span the WTP transfer operations. Data from July 22, 1999, are for a transfer that was made after waste retrieval operations in tank W-10 were $\sim 50 \%$ complete. Data from October 26,1999 , are for a transfer that was made after waste retrieval in tank W-10 was completed, but before any waste had been retrieved from tank W-8. A third data set from March 23, 2000, covers the waste transfer that was made after all the waste had been retrieved from tank W-8. These data indicate that the population (number) distribution of particle sizes tend to center at values under $20 \mu \mathrm{m}$. The cumulative population distribution shown in Fig. 11 indicates that $\sim 99.9+\%$ of the particles possess a characteristic size of $<100 \mu \mathrm{m}$. Both forms of the population distribution indicate a trend toward larger particles over time, especially for particles in the $15-40 \mu \mathrm{m}$ range. 


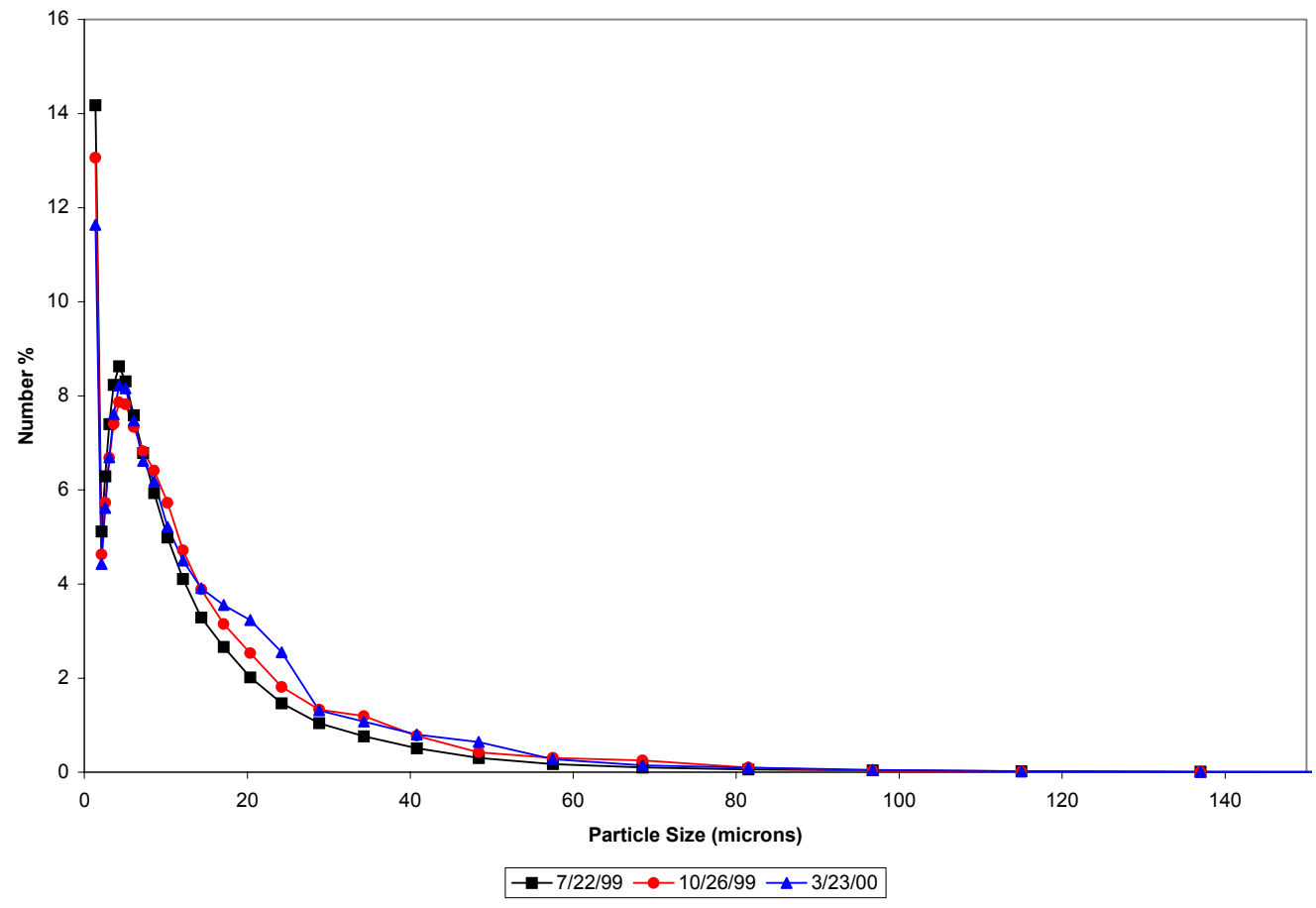

Fig. 10. Particle size distribution by population for selected WTP transfer operations.

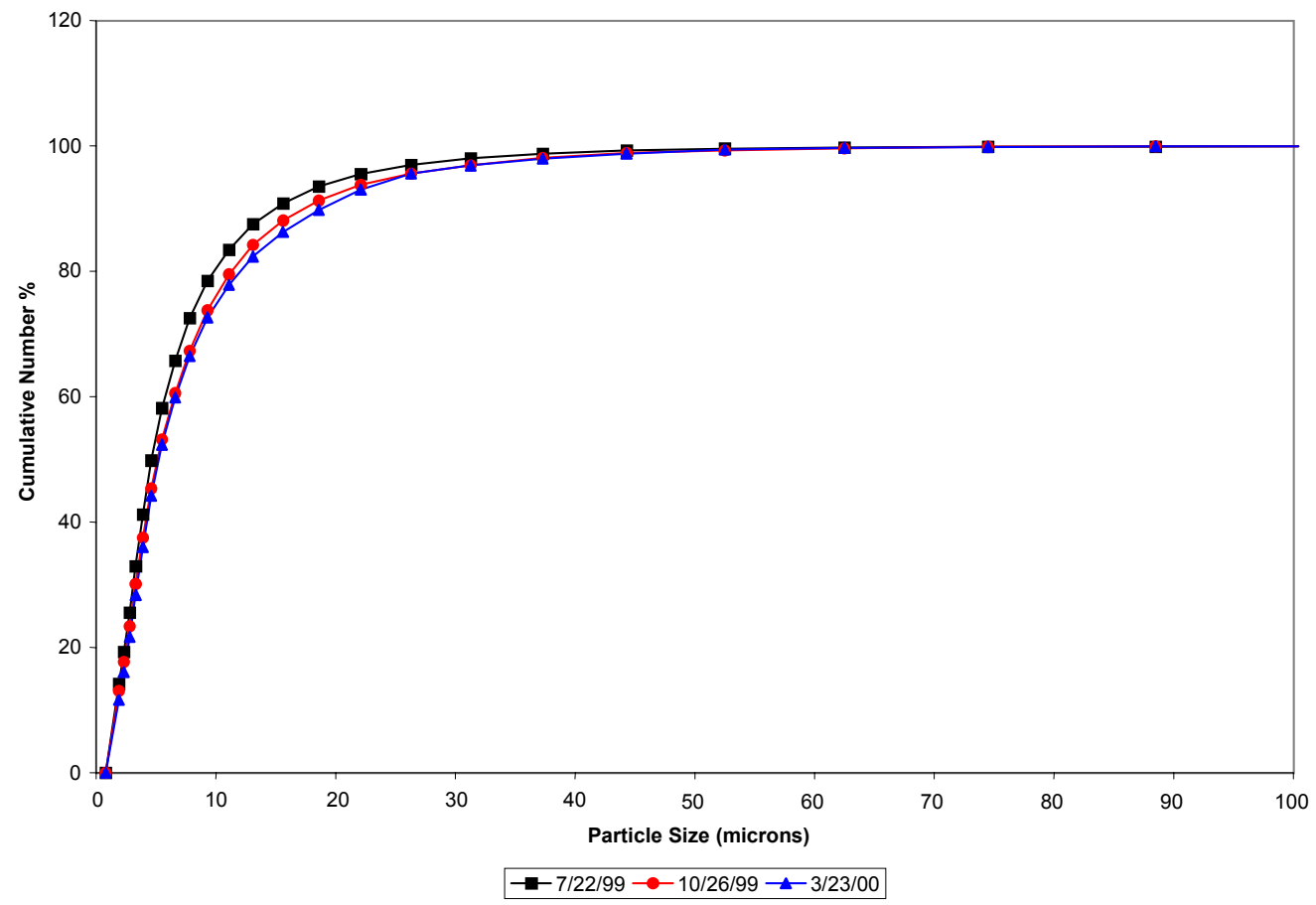

Fig. 11. Cumulative PSD by population for selected WTP transfer operations. 
These population distributions can be put in the form of mass (weight) distributions. The procedure involves multiplying the number in an individual measurement range by the cube of the midpoint of that range's characteristic length, and then dividing the calculated mass in each range by the sum of the masses in all ranges. An implicit assumption in this procedure is that the particles are spherical and of uniform density. Figure 12 shows the resulting weight distribution. The tails of the distribution become more pronounced because of the much greater mass of the larger particles. Changes in the tail of the population distribution, small enough to be masked at the scale at which they were plotted, have a large effect on the mass distribution. The cumulative mass distributions are shown in Fig. 13 and illustrate a significant change over time. Comparing the cumulative population distribution in Fig. 11 with the cumulative weight distribution in Fig. 13, $99.9 \%$ of the particles by number are $<100 \mu \mathrm{m}$ while they account for $\sim 50 \%$ of the mass of distribution. About $96 \%$ of the mass is accounted for by particles $<1000 \mu \mathrm{m}$, which means that $\sim 4 \%$ of the mass is contained in particles $>1 \mathrm{~mm}$ in diameter. On a massaveraged basis, the particle size distribution tends to decrease with time. This is opposite expectations. When particle diameter is the only variable, smaller particles settle more slowly and are easier to keep suspended. It was anticipated that the larger particles would preferentially settle during mixing operations, thereby shifting the particle size distribution in the transferring slurry to larger averages as the tank was emptied. One possible explanation is that the particle density was not uniform, with a portion of the smaller diameter particles being more dense. In this case the smaller, denser particles would preferentially settle during mixing operations and produce the observed results. Another potential cause is that other properties (such as shape or dielectric constant) changed with time, causing particles to stick together into low-density agglomerations. This would cause the laser scanning technique to measure larger-sized particles having densities significantly below that of the crystalline solid.

\subsubsection{Variations in Particle Size Distribution Over One Waste Transfer}

One of the largest waste transfers using the WTP occurred on September 9, 1999, with a total slurry volume of 37,570 gal. The volume of sludge transferred was 5,396 gal and contained a total activity of $6,374 \mathrm{Ci}$, the greatest volume of sludge and total activity transferred in any single WTP transfer operation. Because of the large quantity of sludge transferred, the associated particle size distribution data was selected to ascertain whether measurable changes in the distribution occurred during a single waste transfer. Prior to the transfer, the slurry was recirculated. The duration of the actual transfer was $9 \mathrm{~h}$, but particle size distribution data were logged from 06:44 to 18:40. One-hour segments of the data covering the intervals of 07:00 to 08:00,12:00 to 13:00, and 17:00 to 18:00 were selected to represent particle size distributions at the beginning, midpoint, and end of the transfer. The data within these segments were averaged to obtain unbiased distributions.

The cumulative population distribution at each of the selected intervals is shown in Fig. 14. Over 99.9\% of the particles are $<100 \mu \mathrm{m}$ in characteristic dimension, and $\sim 95 \%$ are $<25 \mu \mathrm{m}$. Variations in the population distribution are negligible. 


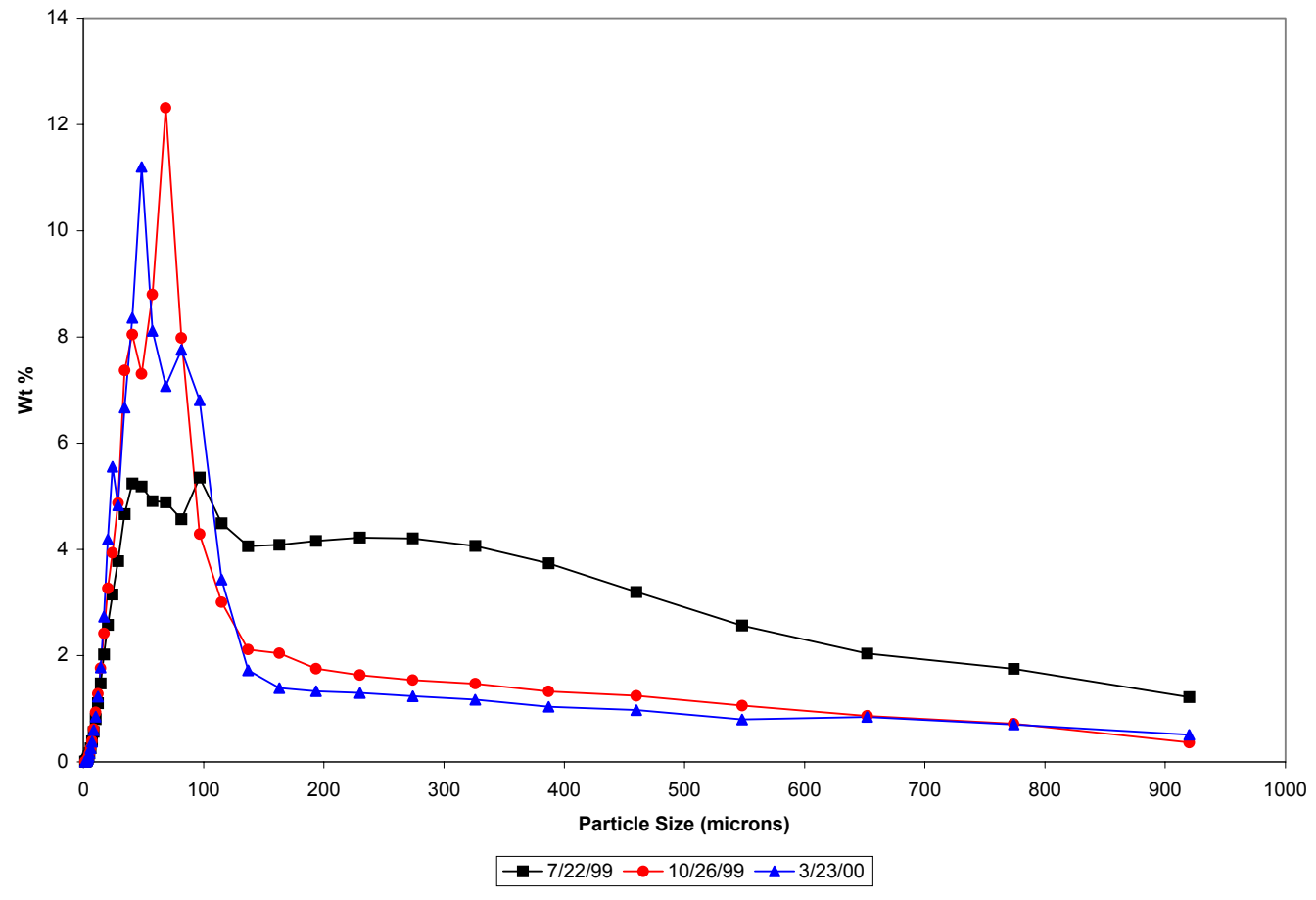

Fig. 12. Particle size distribution by weight for selected WTP transfer operations.

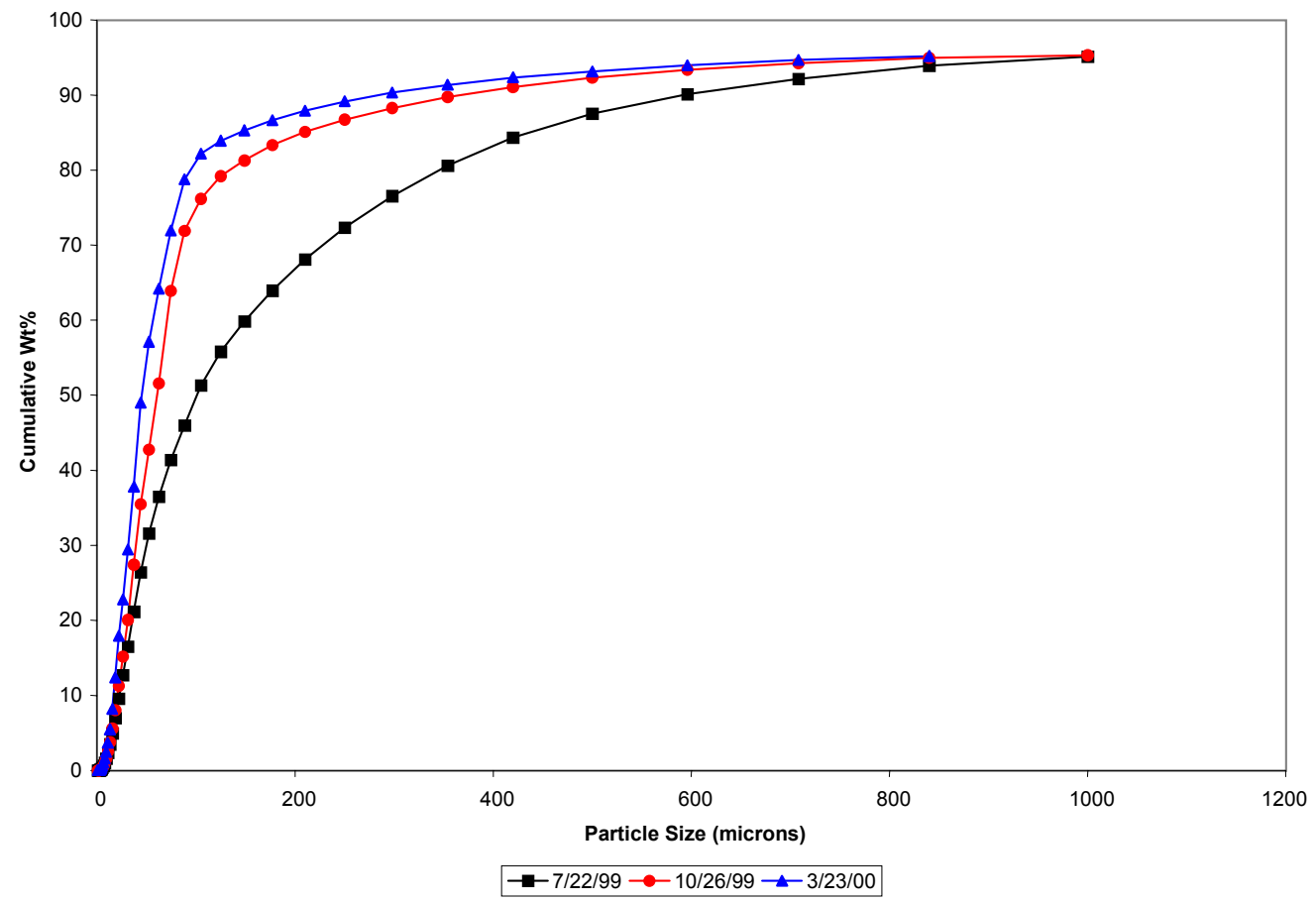

Fig. 13. Cumulative PSD by weight for selected WTP transfer operations. 


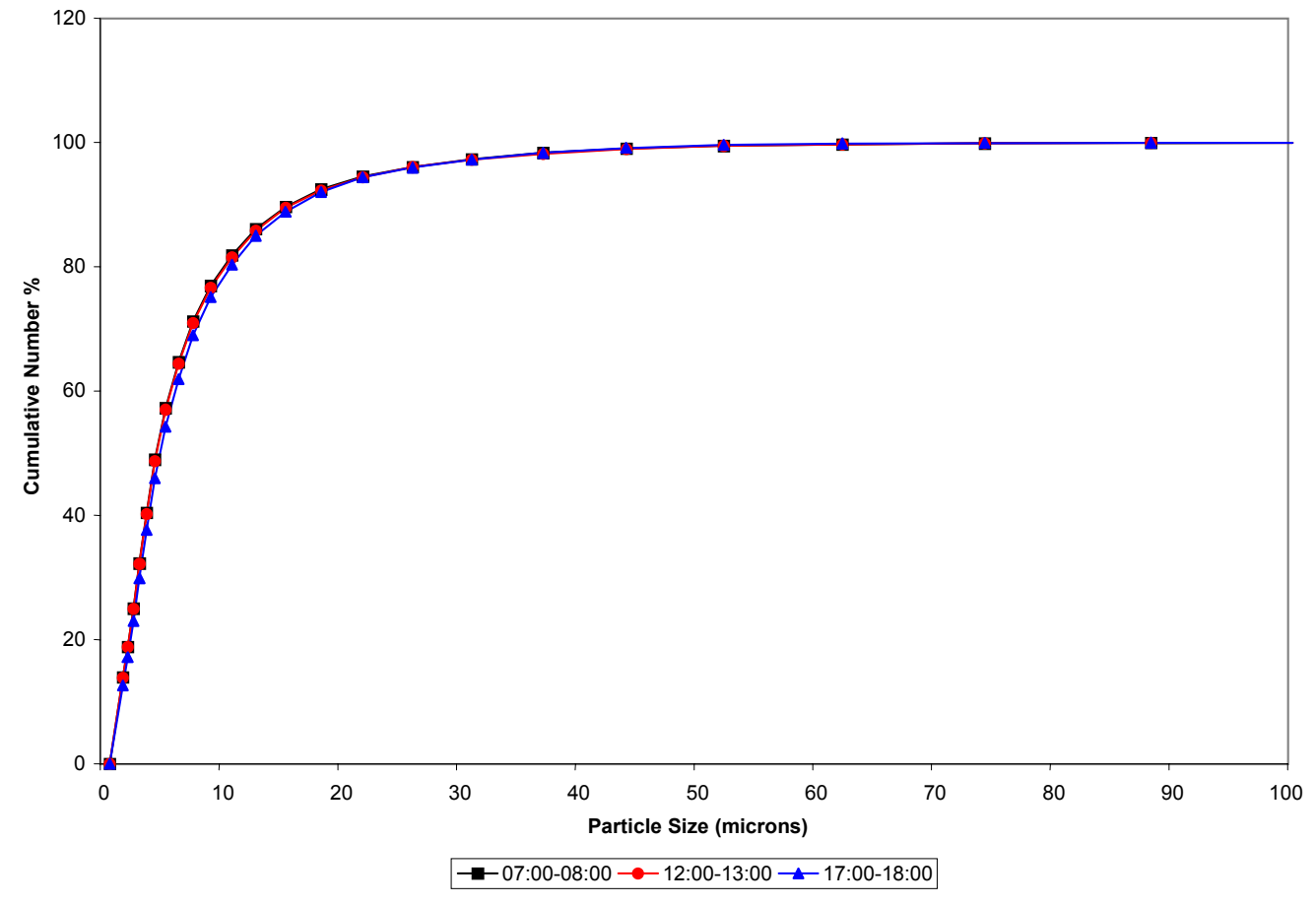

Fig. 14. Cumulative PSD by number for the waste transfer of September 9, 1999.

It was previously found that the weight distribution data reveal details that the population distribution does not. Thus, the data were processed to obtain a cumulative particle size distribution by weight as shown in Fig. 15. Although a small decrease in the particle size appears to occur during the middle part of the waste transfer, the small magnitude of the decrease indicates that it is not significant. These results provide confidence that daily averages in the particle size distribution data are sufficient to track longterm changes and to characterize the entire transfer.

\subsubsection{Effect of Installing the Flygt Mixer on Particle Size Distribution}

One of the two Flygt mixers from tank W-5 was installed in tank W-9 on August 13, 1999. Because of routine shakedown, including adjustments and tests to verify the capability to meet the WAC, the unit was not used to mix the slurry during a transfer operation until September 28, 1999. The last transfer of waste to occur prior to use of the Flygt mixer took place on September 9, 1999. Particle-size distribution data obtained on these two dates were examined to ascertain the effect, if any, that use of the Flygt mixer had on the distribution. Particle-size distributions averaged over the duration of each waste transfer were taken to be representative of overall changes.

The cumulative population distributions measured on September 9, 1999, and September 28, 1999, are compared in Fig. 16. These data show very little variation in the distributions, indicating a small effect caused by the use of the Flygt mixer. Again, the data were transformed to a cumulative weight distribution as shown in Fig. 17. These data show that the distribution following installation of the Flygt mixer has a more persistent tail, indicating a trend to maintain larger particles in suspension. Overall, the variation is small and may not be significant. 


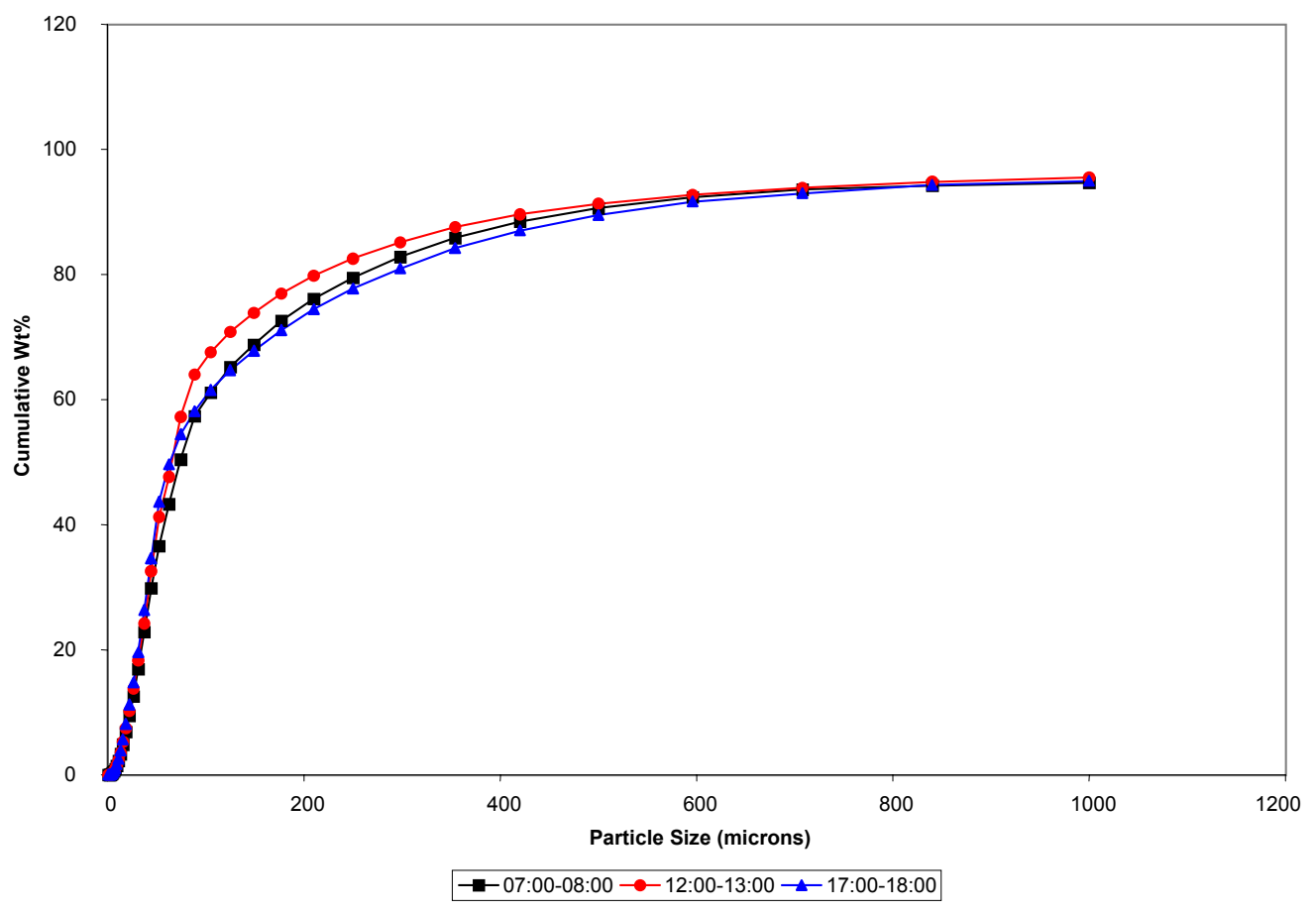

Fig. 15. Cumulative PSD by weight for the waste transfer of September 9, 1999.

\subsubsection{Settling Data on Sludge Remaining in Tank W-9 at Completion}

Figure 18 shows the settling rate of sludge remaining in tank W-9 after the completion of the MVST transfers. This analysis was performed by the ORNL Radioactive Materials Analytical Laboratory. The procedure included mixing a $20 \mathrm{~mL}$ aliquot of the sludge in a 1:5 ratio with $4 \mathrm{MNaOH}$ solution to produce $100 \mathrm{~mL}$ of slurry. After $1 \mathrm{~min}$ of mixing, the slurry was allowed to stand for $24 \mathrm{~h}$ in a $100-\mathrm{mL}$ graduated cylinder (which is $18 \mathrm{~cm}$ tall from the 0 to $100 \mathrm{~mL}$ marks). The height of the sludge layer, as indicated by the milliliter markings on the cylinder, was recorded at various times during the settling period. The raw data were converted to liquid/solid phase heights and are shown in Fig. 18. The settling rate is roughly consistent with Stokes' law for sludge systems similar to this one (Giaquinto, Keller, and Mills, 1997). Particle-particle interactions (hindered settling) and particle drag against the cylinder walls cause deviations from theory and become more prominent as settling progresses. The initial slope of the settling curve indicates a settling rate of $4 \mathrm{~cm} / \mathrm{h}$. This is consistent with solid particles of $5 \mathrm{sm}$ diam and density of $2 \mathrm{~g} / \mathrm{cm}^{3}$ settling in a fluid having the density and viscosity of water at room temperature. Generally, the raw data are used as an empirical guide in estimating settling time. For example, the data indicate that the slurry settles to $\sim 60 \%$ of its initial height in $400 \mathrm{~min}$, and that settling to $\sim 50 \%$ of the initial height requires $1200 \mathrm{~min}$. 


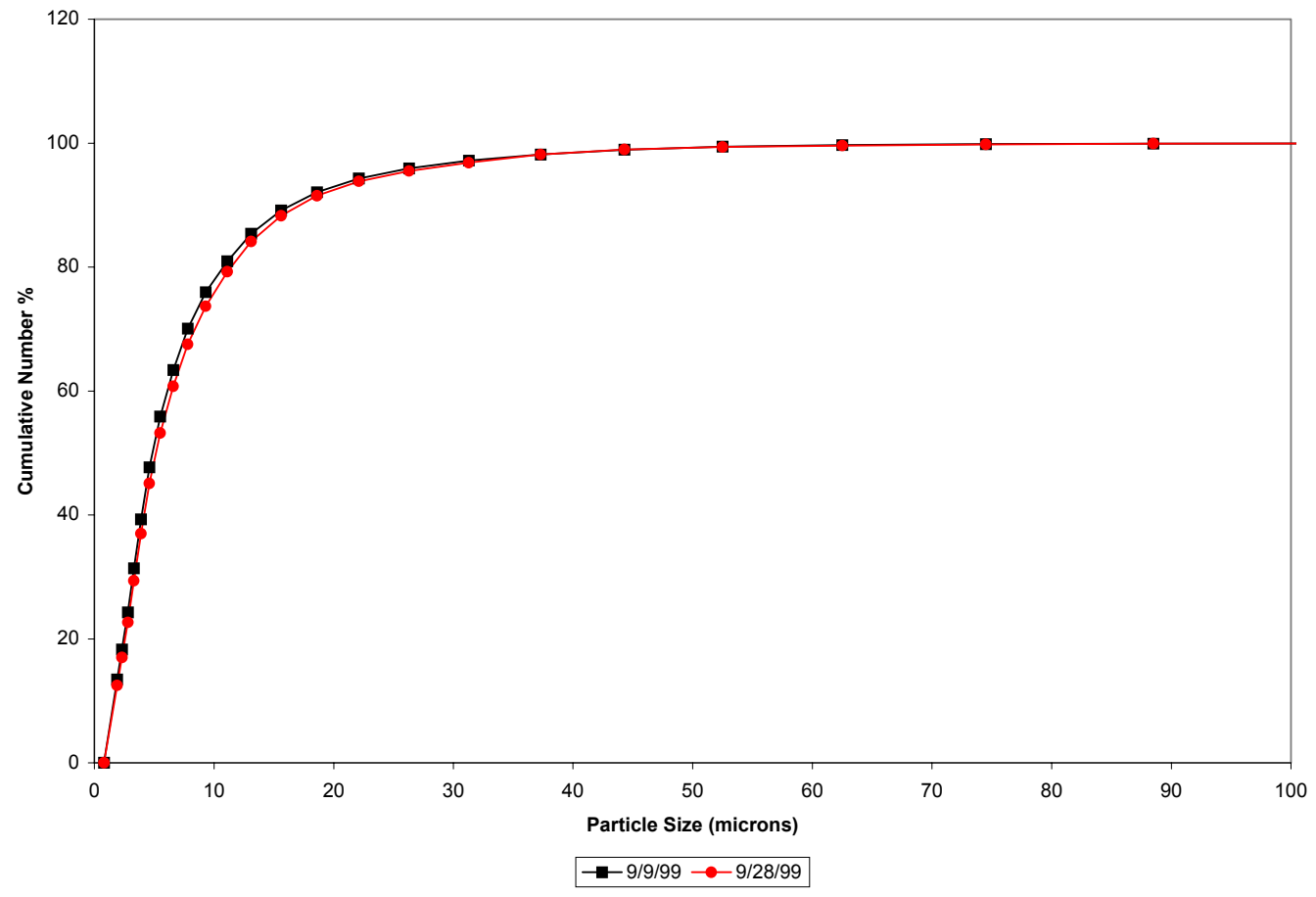

Fig. 16. The effect of the Flygt mixer on cumulative PSD by population.

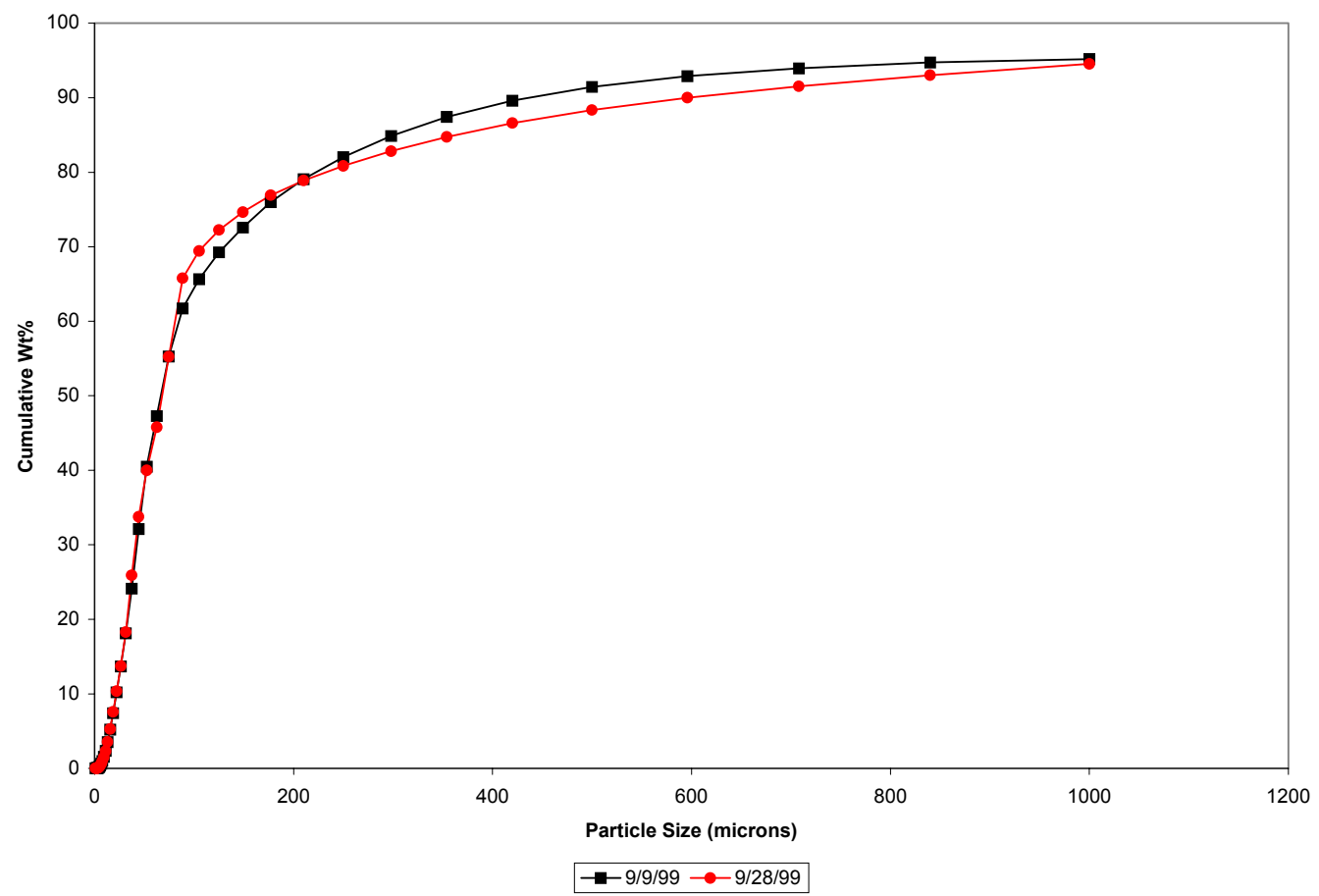

Fig. 17. The effect of the Flygt mixer on cumulative PSD by weight. 


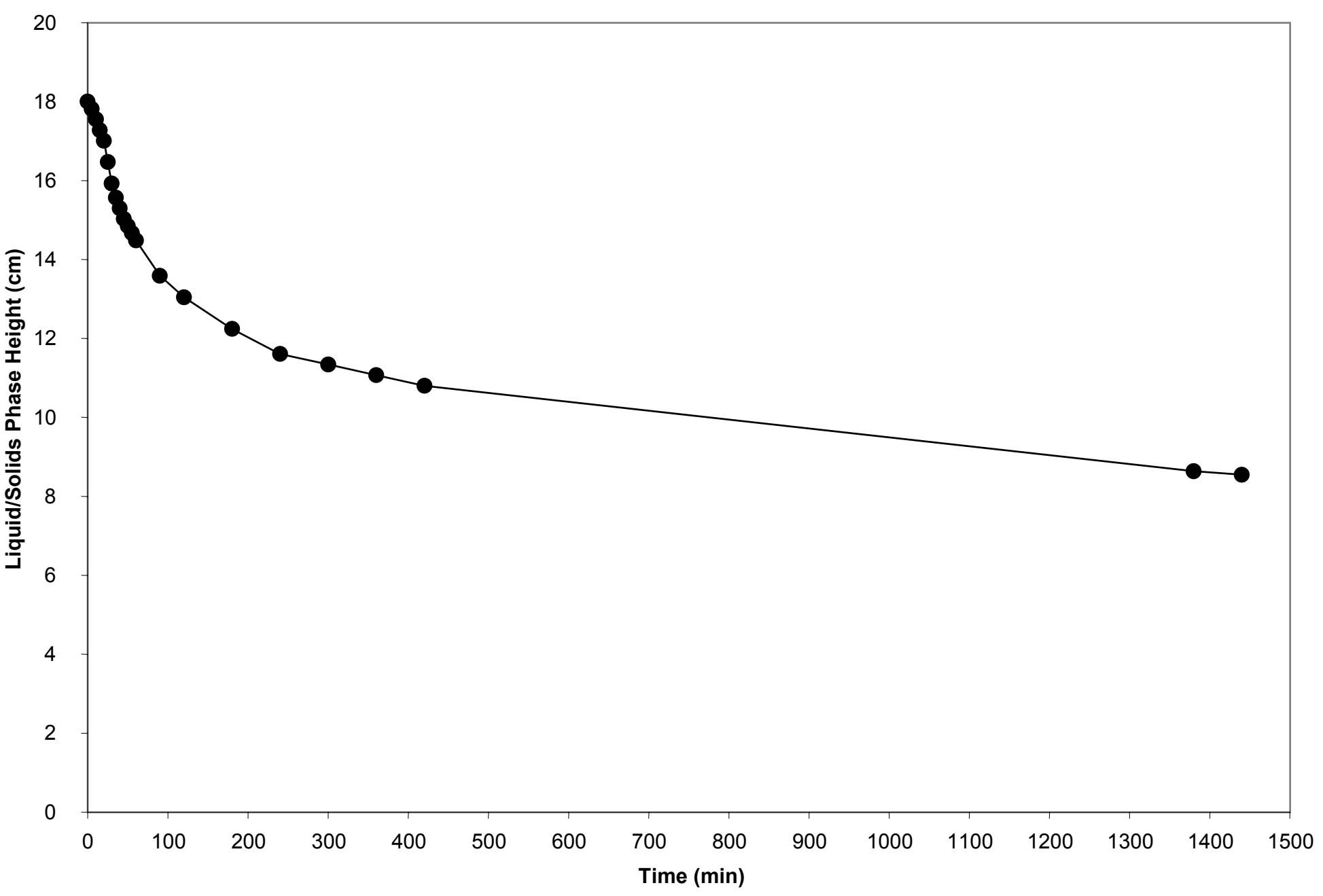

Fig. 18. Settling rate of W-9 sludge after completion of MVST transfers, June 28, 2000. Starting sludge volume is about $20 \mathrm{~mL}$. 


\subsection{WaRTS Transfers to W-23}

A total of 14 transfers were made to tank W-23 while WaRTS was operational in tank W-9. These batch transfers were always significantly smaller than the Discflo batch transfers because the WaRTS could not maintain the design flow rate while sluicing, and the WaRTS operated only while the in-tank robotic systems were actively sluicing. Table 4 displays information on the WaRTS transfers.

Table 4. Operating summary of WaRTS transfers to tank W-23

\begin{tabular}{|c|c|c|c|c|}
\hline \multicolumn{2}{|c|}{ Transfer } & \multirow{2}{*}{$\begin{array}{c}\text { Volume } \\
\text { (gal) }\end{array}$} & \multirow{2}{*}{$\begin{array}{l}\text { Est. sludge volume } \\
\text { (gal) }{ }^{l}\end{array}$} & \multirow{2}{*}{$\begin{array}{c}\text { Particles of size }<105 \mu \mathrm{m} \\
(\%)^{2}\end{array}$} \\
\hline No. & Date(s) & & & \\
\hline 1 & $07 / 13 / 00$ & 8,850 & 1,372 & Not available \\
\hline 2 & $07 / 18 / 00$ & 10,500 & 1,550 & 99.970 \\
\hline 3 & $07 / 22 / 00$ & 17,450 & 2,514 & 99.963 \\
\hline 4 & $07 / 26-27 / 00$ & 24,100 & 5,297 & 99.933 \\
\hline 5 & $08 / 01-02 / 00$ & 18,900 & 3,293 & 99.892 \\
\hline 6 & $08 / 15-16 / 00$ & 19,450 & 5,425 & 99.827 \\
\hline 7 & 08/19-21/00 & 19,200 & 5,208 & 99.735 \\
\hline 8 & 08/29-30/00 & 17,950 & 2,816 & 99.842 \\
\hline 9 & 09/06-07/00 & 10,500 & 787 & 99.881 \\
\hline Tot: & & 146,900 & 28,262 & \\
\hline
\end{tabular}

${ }^{I}$ The initial total activity in both sludge and supernatant was estimated at $85 \times 10^{3} \mathrm{Ci}$. Approximately $(7-10) \times 10^{3} \mathrm{Ci}$ contributed by residual sludge and contamination trapped in the tank walls was estimated to remain in the tank. Thus, $\geq 75 \times 10^{3} \mathrm{Ci}$ was removed from the tank.

${ }^{2}$ Percent by count, from LasenTec measurements.

Figure 19 displays the particle size distribution as measured by the LasenTec instrument for the July 22, 2000, slurry transfer from tank W-9 to tank W-23 in the ORNL active LLLW system. This was the second of 14 transfers from W-9 to W-23 using the WaRTS. Choppy variations occurred in the count of all particles and in the smallest particle size range. The contrast with a relatively smooth total count line in Fig. 7 illustrates a "WaRTS effect." One possible explanation of the difference lies in the way the two systems were designed and operated. The Discflo system was intended to achieve and maintain a relatively steady flow and pressure while WaRTS is a surge tank based system, which by design, fills, empties, and refills during transfers, making the flow rate oscillate. Operating in conjunction with the WaRTS SPS, full-pipe flow to the inlet of the diaphragm pump is maintained. Thus, the intermittent flow pattern of the confined sluicing end-effector (CSEE) jet pump discharge is accommodated but the solids concentration profile retains artifacts of the oscillatory flow.

An approach similar to that described in Sect. 6.1.1 was used to screen the data for long-term changes in the particle-size distribution. Three transfer campaigns were selected to represent conditions at the beginning, midpoint, and endpoint of the waste transfer operations using the WaRTS. The dates of these transfers were July 18, 2000, August 2, 2000, and August 30, 2000, respectively. Average particle size distribution data over the entire transfer was taken as representative of the waste transferred on a particular day. 


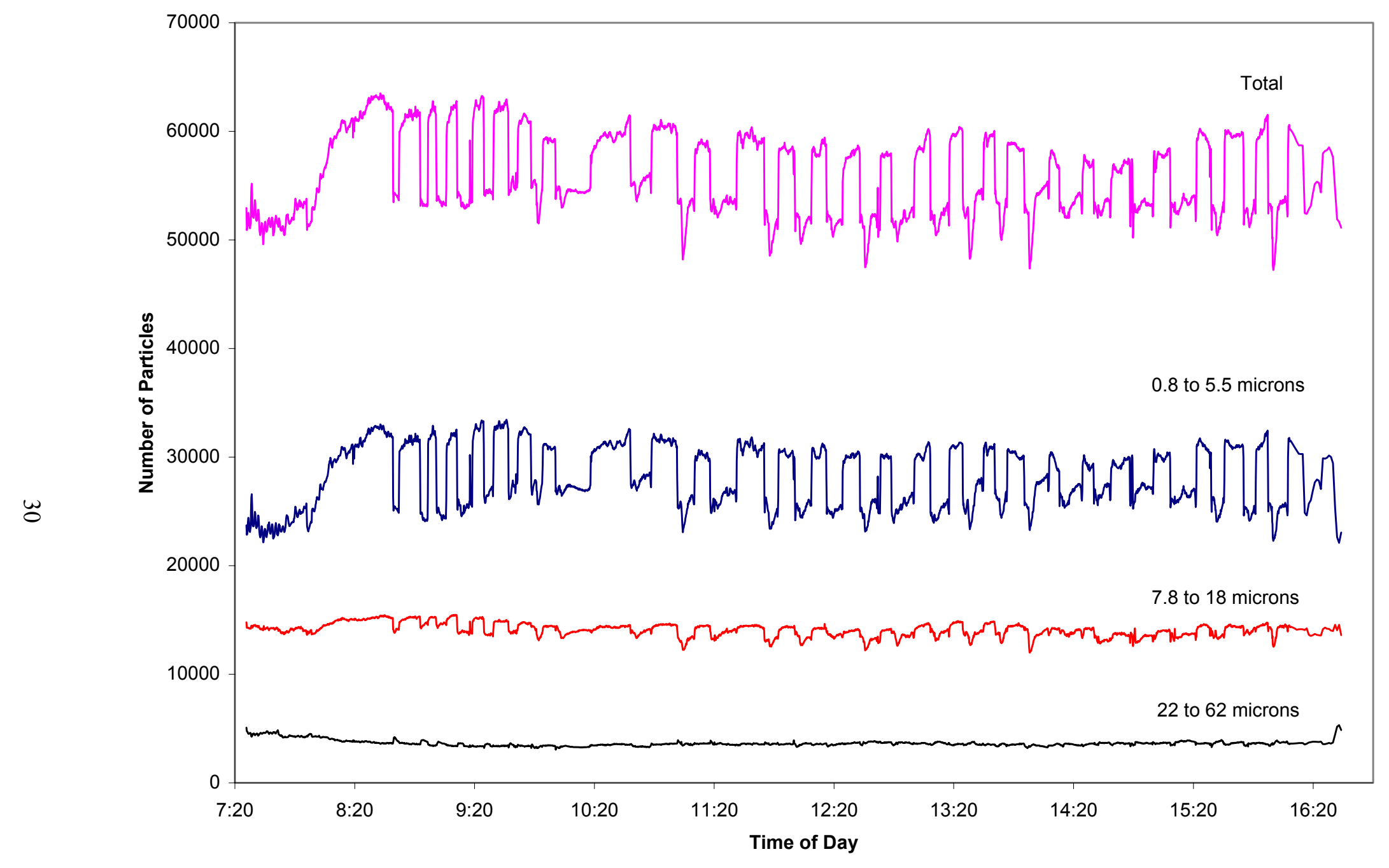

Fig. 19. The number of particles measured by the LasenTec instrument during the WaRTS slurry transfer on July 22, 2000 (tank W-9 to ORNL active LLLW tank W-23). 
Figure 20 shows the population distribution of particle sizes. Variations in these distributions are small. These variations are more clearly shown in the weight distribution shown in Fig. 21 or the cumulative weight distribution shown in Fig. 22. Overall, the weighted particle size distribution is broader at the midpoint of the WaRTS operations. The mean particle sizes were smaller at the beginning of the WaRTS as expected, but do not monotonically increase throughout the duration of the WaRTS operation. The combination of the fill-refill cycling of WaRTS and potential variations in particle density and shape may explain the complex behavior observed.

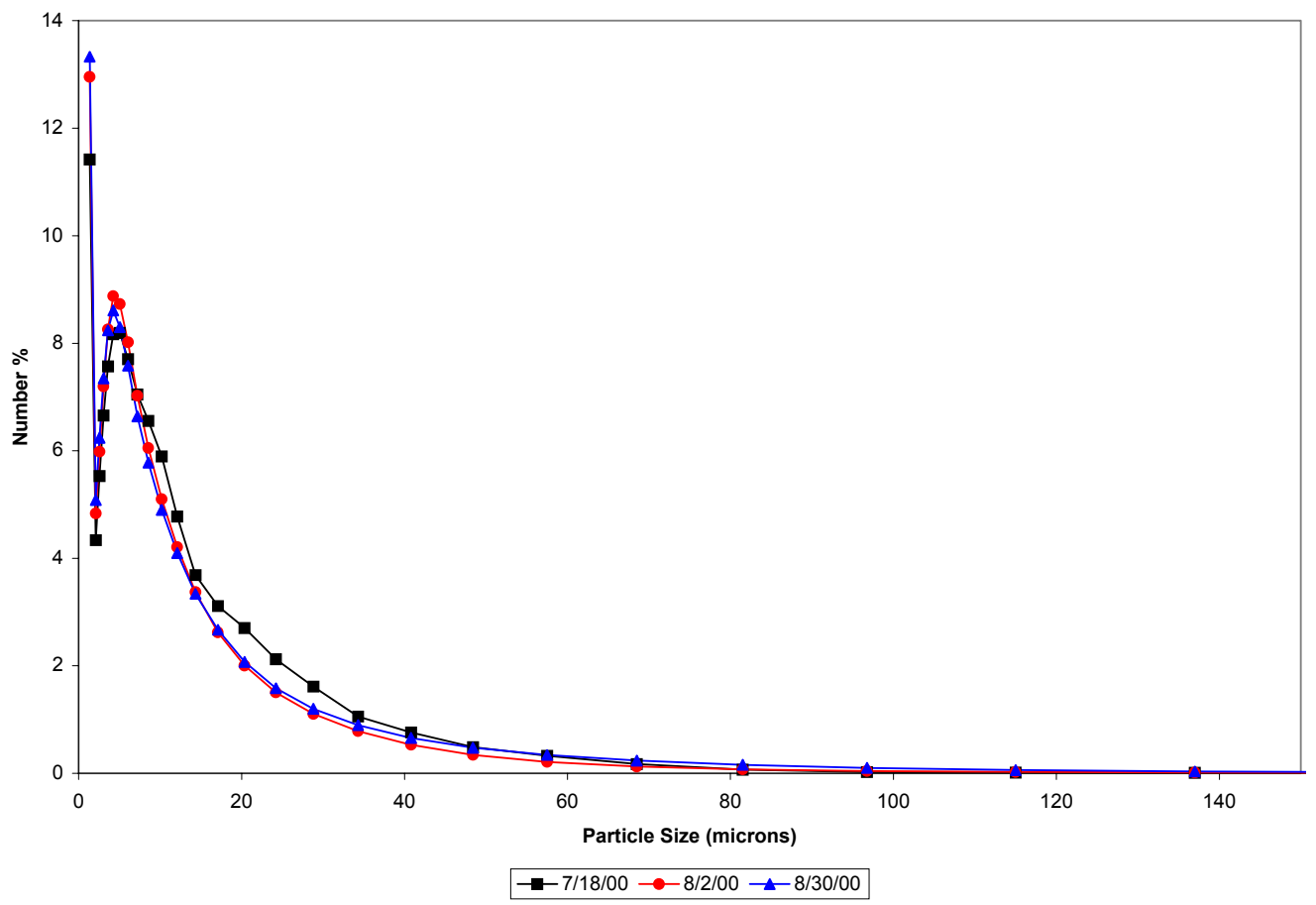

Fig. 20. PSD by number for selected transfers using WaRTS. 


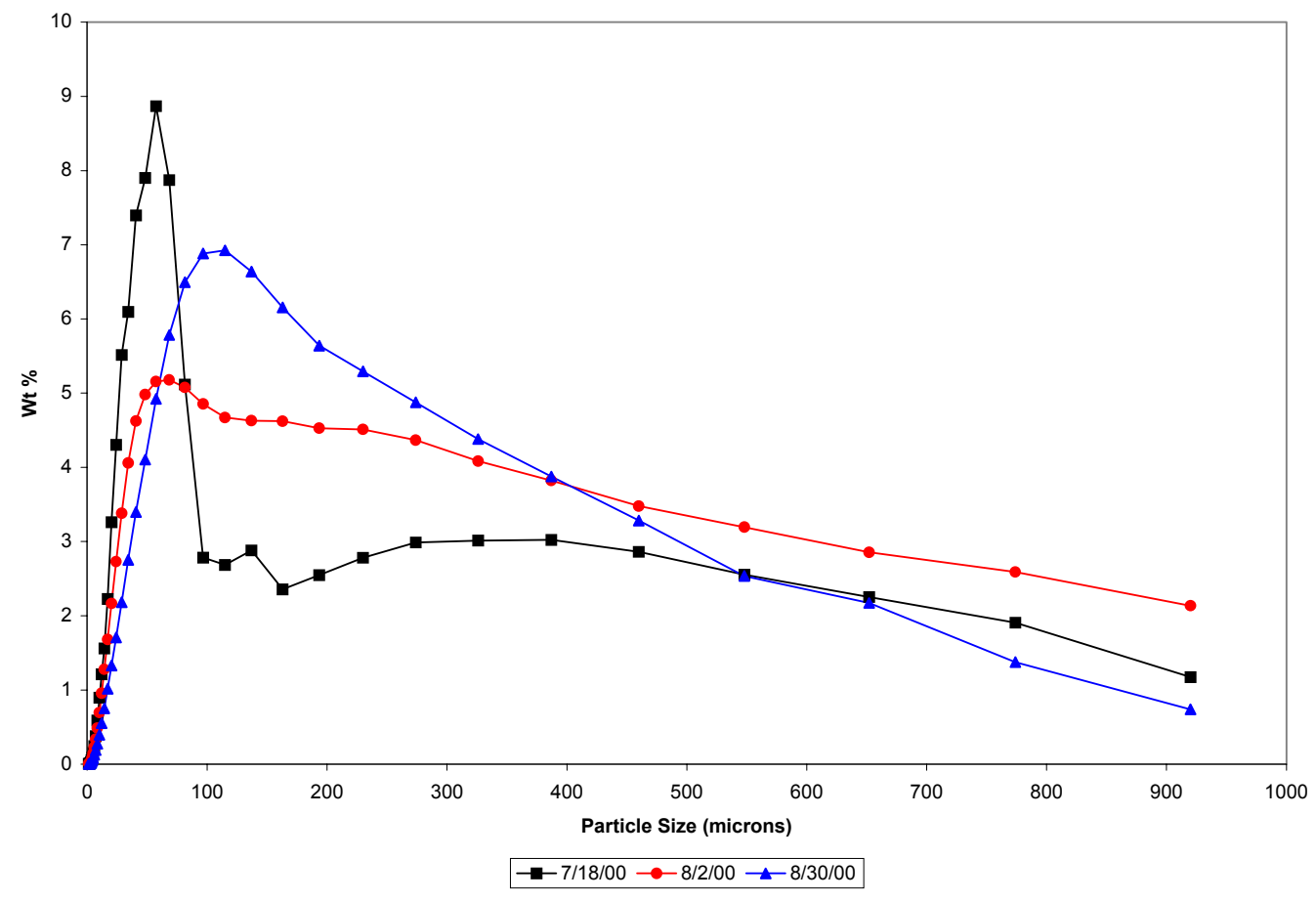

Fig. 21. PSD by weight for selected transfers using WaRTS.

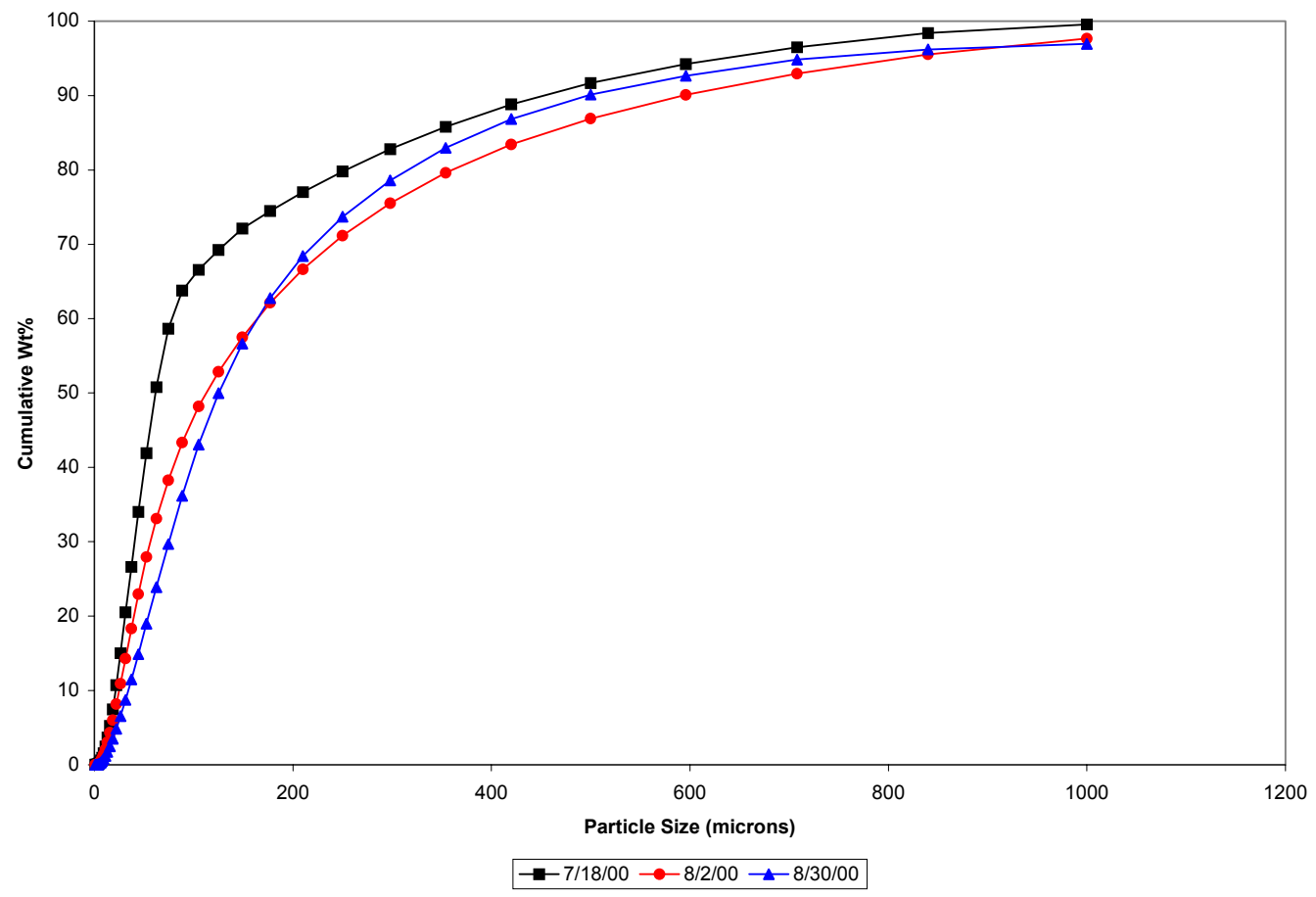

Fig. 22. Cumulative PSD by weight for selected transfers using WaRTS. 


\subsection{TH-4 TRANSFERS TO W-23}

Transfer operations from tank TH-4 were completed in a brief campaign on January 13-15, 2001. Each day, one transfer was made following a period of mixing by the Russian PMP. The PMP was kept running while the tank was being emptied. A diaphragm pump was used to transfer the slurry through a doubly contained, above ground temporary pipeline to the PCS enclosure in the STF. This temporary pipeline from TH-4 to the PCS was composed of flexible hose inside hard PVC pipe. From there it entered the doubly contained, underground steel piping, which was used during the two previous phases of waste retrieval to transfer the waste from tank W-9 to tank W-23.

The initial sludge content of tank TH-4 was estimated at 6,266 gal (Autry et al., 1990). Supernatant was recycled from tank W-8 to provide a sluicing medium. During sluicing and sludge removal operations, a total of over 24,000 gal of slurry was transferred from tank TH-4 to tank W-23. Residual sludge was estimated at a volume of 1,098 gal, indicating a sludge removal of $\sim 82 \%$. The total activity of waste remaining in the tank was reduced from $\sim 3.37 \mathrm{Ci}$ to $\sim 0.59 \mathrm{Ci}$.

\subsubsection{Variation in Particle Size Distribution}

Figure 23 displays the particle size distribution as measured by the LasenTec instrument for the January 13, 2001, slurry transfer from tank TH-4 to tank W-23 in the ORNL active LLLW system. This was the first of three transfers from TH-4 to W-23, which used the Russian PMP to mix the contents of the tank. The large spike at midday is apparently due to the restarting of the PMP mixer, as recorded in the shift supervisor's log.

The precipitous decrease in the count rate that occurred in the early afternoon $(\sim 13: 30)$ indicates that the concentration of undissolved solids in the slurry decreased significantly. The reason for this is unclear. Particle-size distributions are more clearly presented in terms of number percent and weight percent as discussed in Sect. 6. The available data were averaged over the time intervals of interest to obtain distributions for which measurement error was dampened. Data in time intervals of 11:00-12:00 and 14:00-15:00 were taken to represent the particle distributions before and after the rapid-change event. Figures 24 and 25 show the population distribution and weight percent distribution, respectively. The population distribution indicates a trend to larger-sized particles and a broader range of particle sizes. This is consistent with the presumption that smaller, more readily suspended solid particles would be removed from the tank earlier. On the basis of weight fraction, shown in Fig. 25, the distribution obtained in the morning shows one peak at $\sim 60 \mu \mathrm{m}$. Two peaks occur in the afternoon distribution, one at $\sim 100 \mu \mathrm{m}$ and another at $\sim 200 \mu \mathrm{m}$. Another change between the two curves is that the distribution obtained in the afternoon is much noisier than the distribution measured before the midday spike. This is more clearly shown in the cumulative weight fraction distribution of Fig. 26. The change in the distribution might be an artifact of the PMP restart, exacerbated by a change in the liquid flow rate (i.e., increased dilution), or a problem with the LasenTec instrument. 


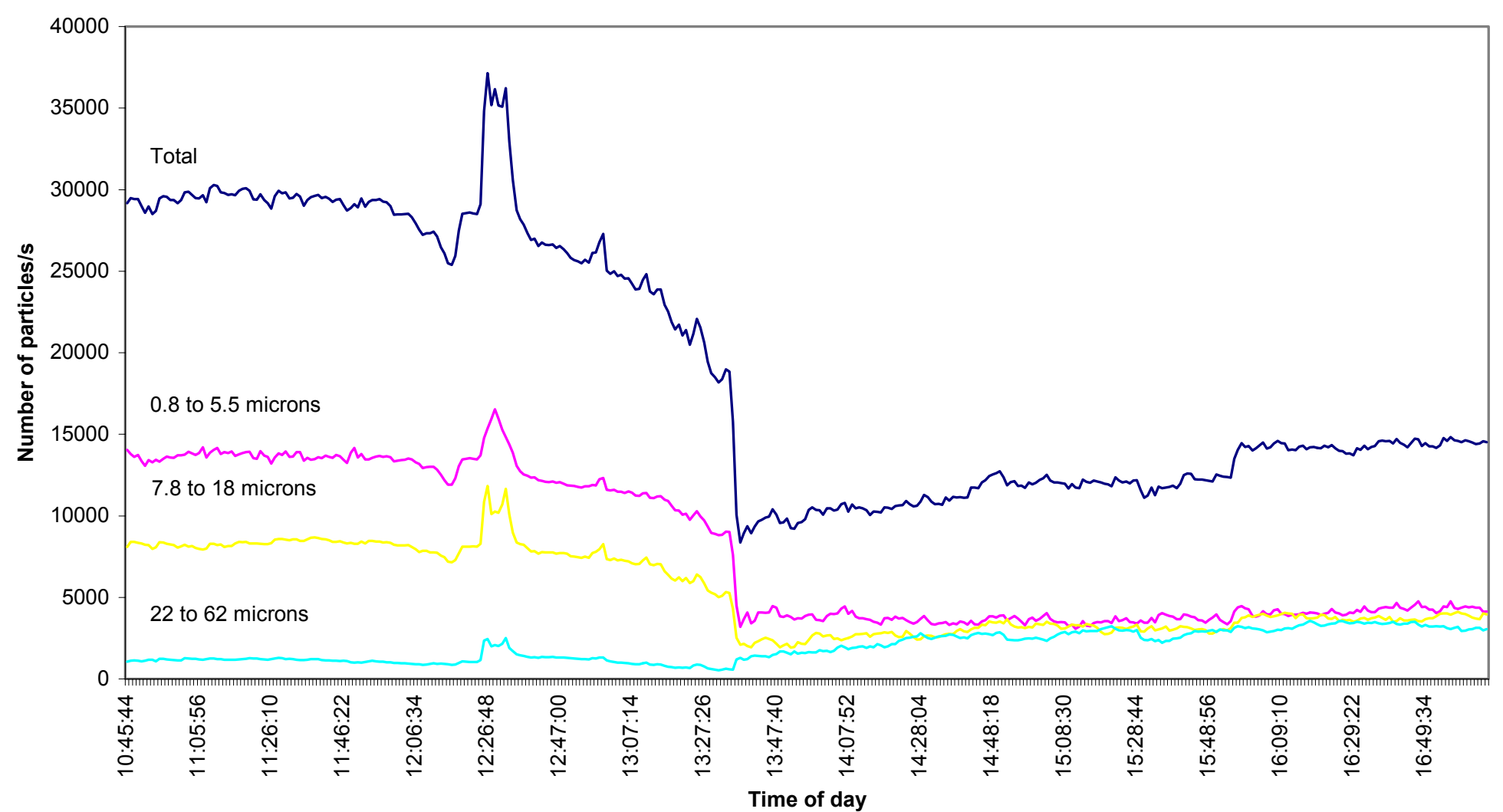

Fig. 23. The number of particles measured by the LasenTec instrument during the Russian PMP slurry transfer from tank TH-4 to ORNL active LLLW tank W-23, January 13, 2001. Range of particle size is measured in microns. 


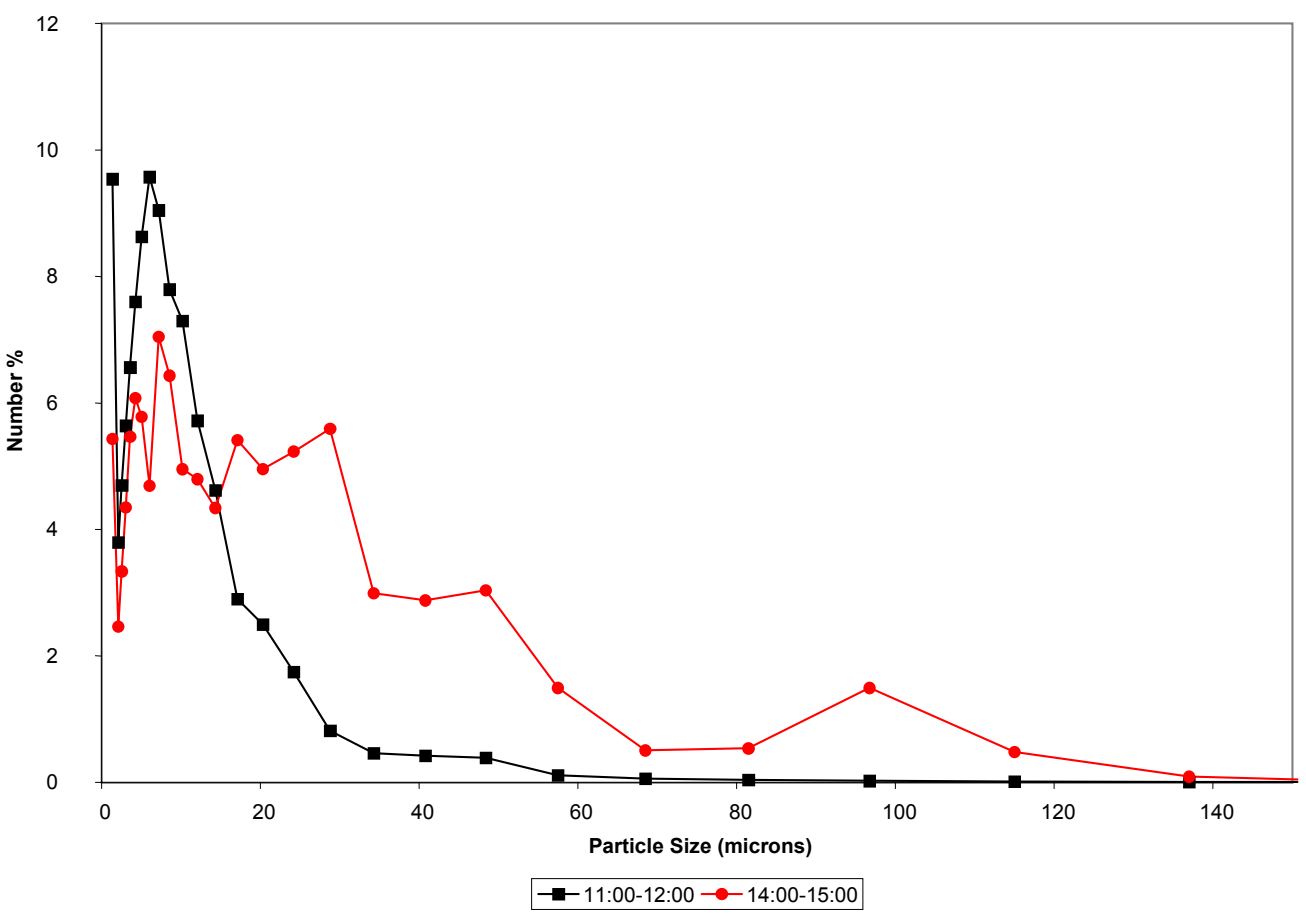

Fig. 24. Particle-size distribution by population for transfers from TH-4 on January 13, 2001.

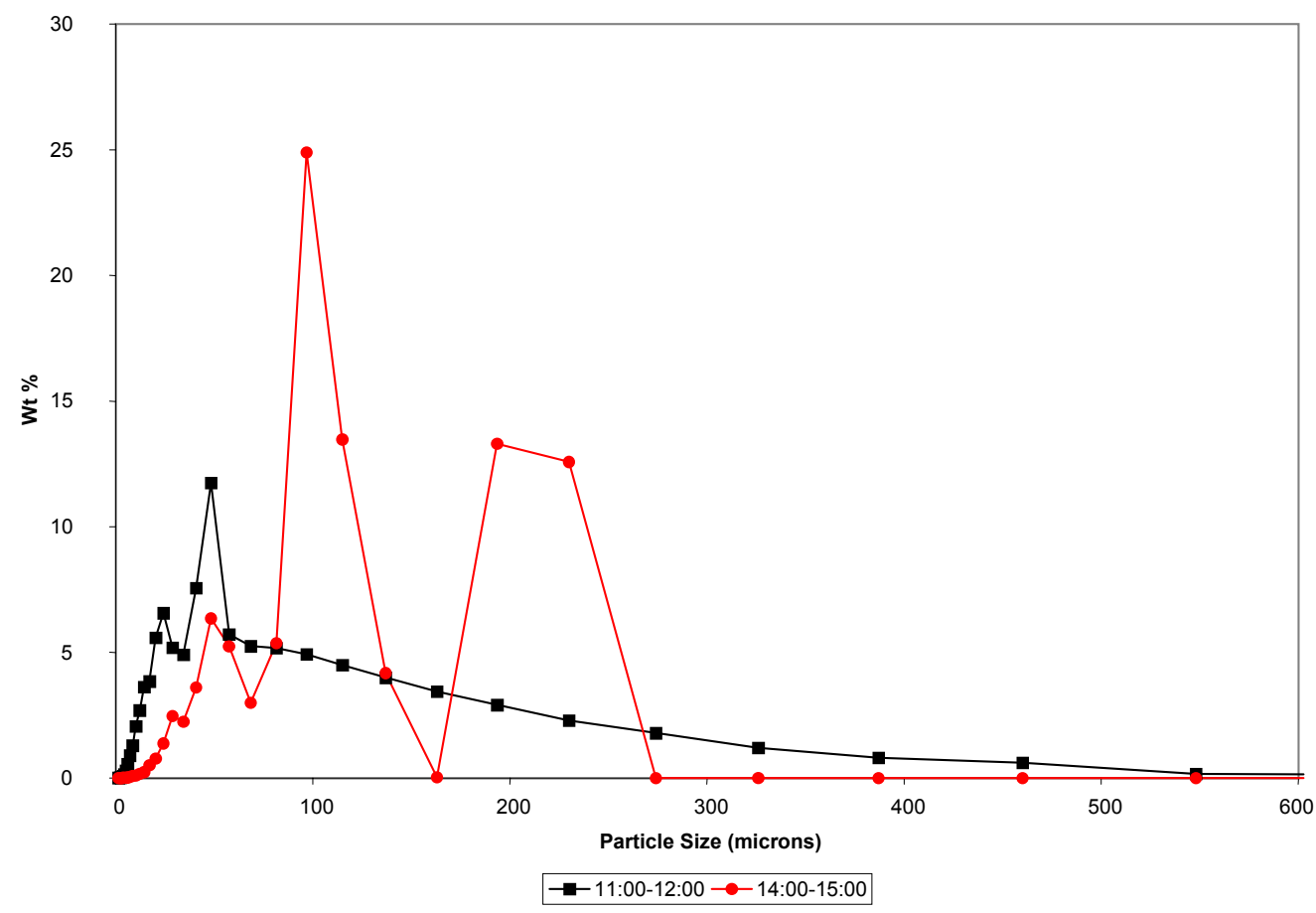

Fig. 25. Particle-size distribution by weight for transfers from TH-4 on January 13, 2001. 


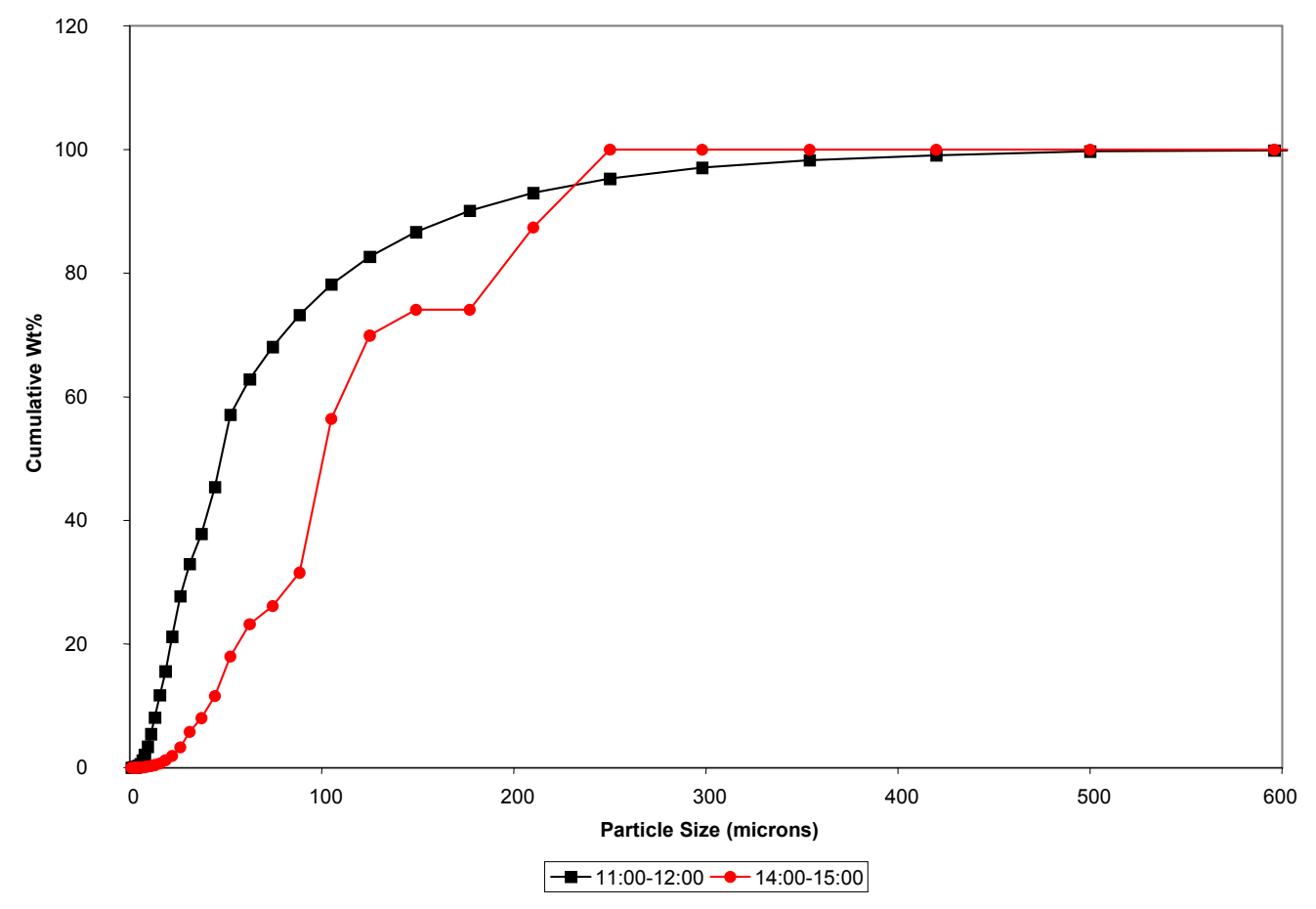

Fig. 26. Cumulative PSD by weight for transfers from TH-4 on January 13, 2001.

Data collected during operations on the following two days were examined for overall trends. Although occasional spikes in the LasenTec data occurred, no lasting changes of significance took place following the spikes. Changes in the particle sizes over the course of waste transfer from tank TH-4 to tank W-23 are illustrated by comparing data from near the beginning and end of the retrieval operations as follows. Data averaged over two 1-h intervals; namely, 11:00-12:00 on January 13, 2001, and 15:00-16:00 on January 15,2001 , were selected because the equipment was operating smoothly during these times. The population and weight distributions for these intervals are shown in Figs. 27 and 28. Both show a general broadening of the particle-size distribution and a shift toward larger particle sizes. The weight distribution in Fig. 28 shows larger particles persist until near the end of the transfer operation. Figure 29 illustrates the cumulative weight percent distribution for these two intervals from which the same conclusions can be drawn.

\subsubsection{Settling Data on Tank W-23 Sludge}

Raw data on the settling rate of sludge collected from tank W-23 is shown in Fig. 30. This data may be treated as described in Sect. 6.1.4. The data indicate that the slurry settles to $\sim 50 \%$ of its initial height in $15 \mathrm{~min}$, and that settling to $\sim 20 \%$ of the initial height requires $60 \mathrm{~min}$. 


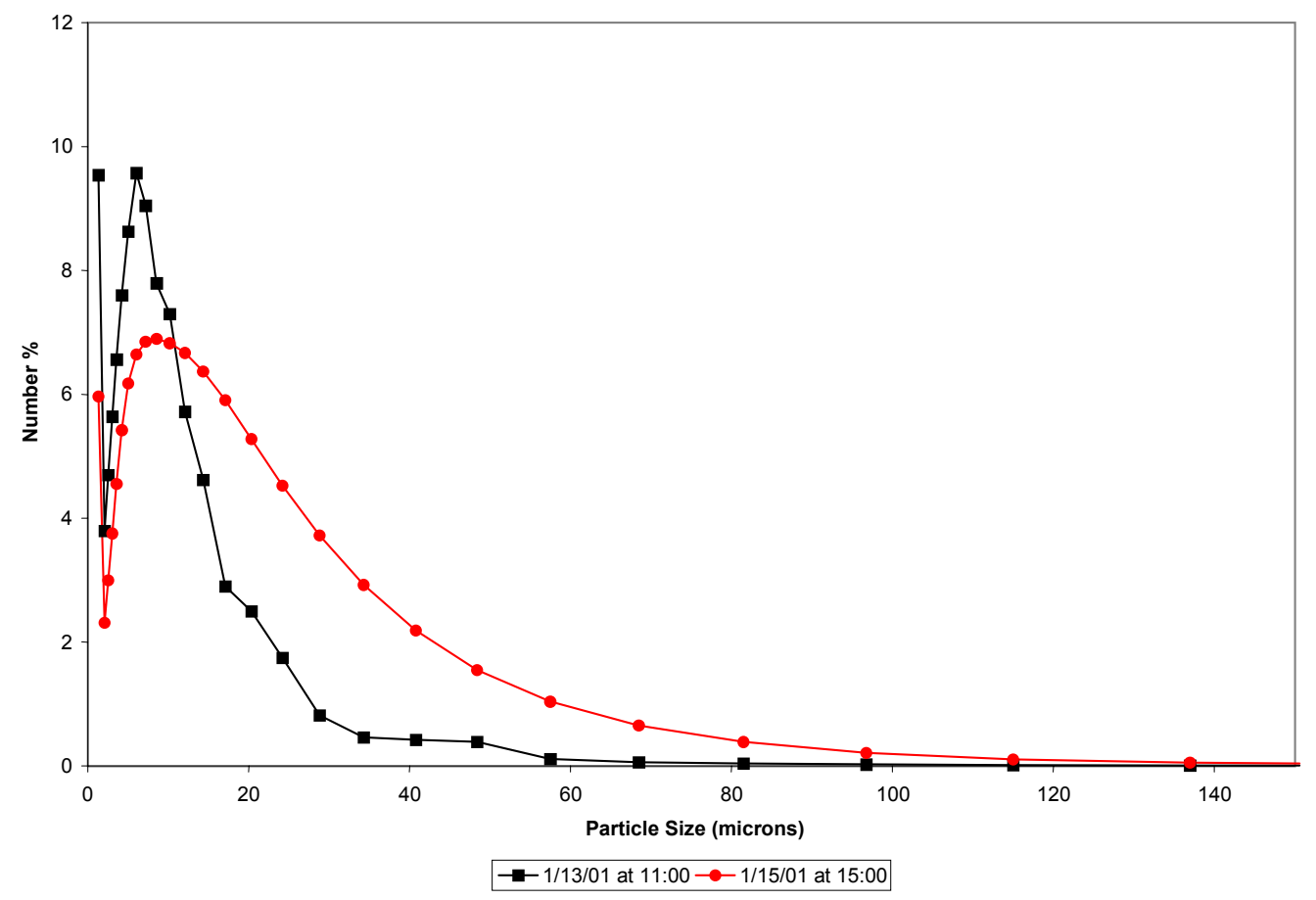

Fig. 27. PSD by population for selected transfers of waste from tank TH-4.

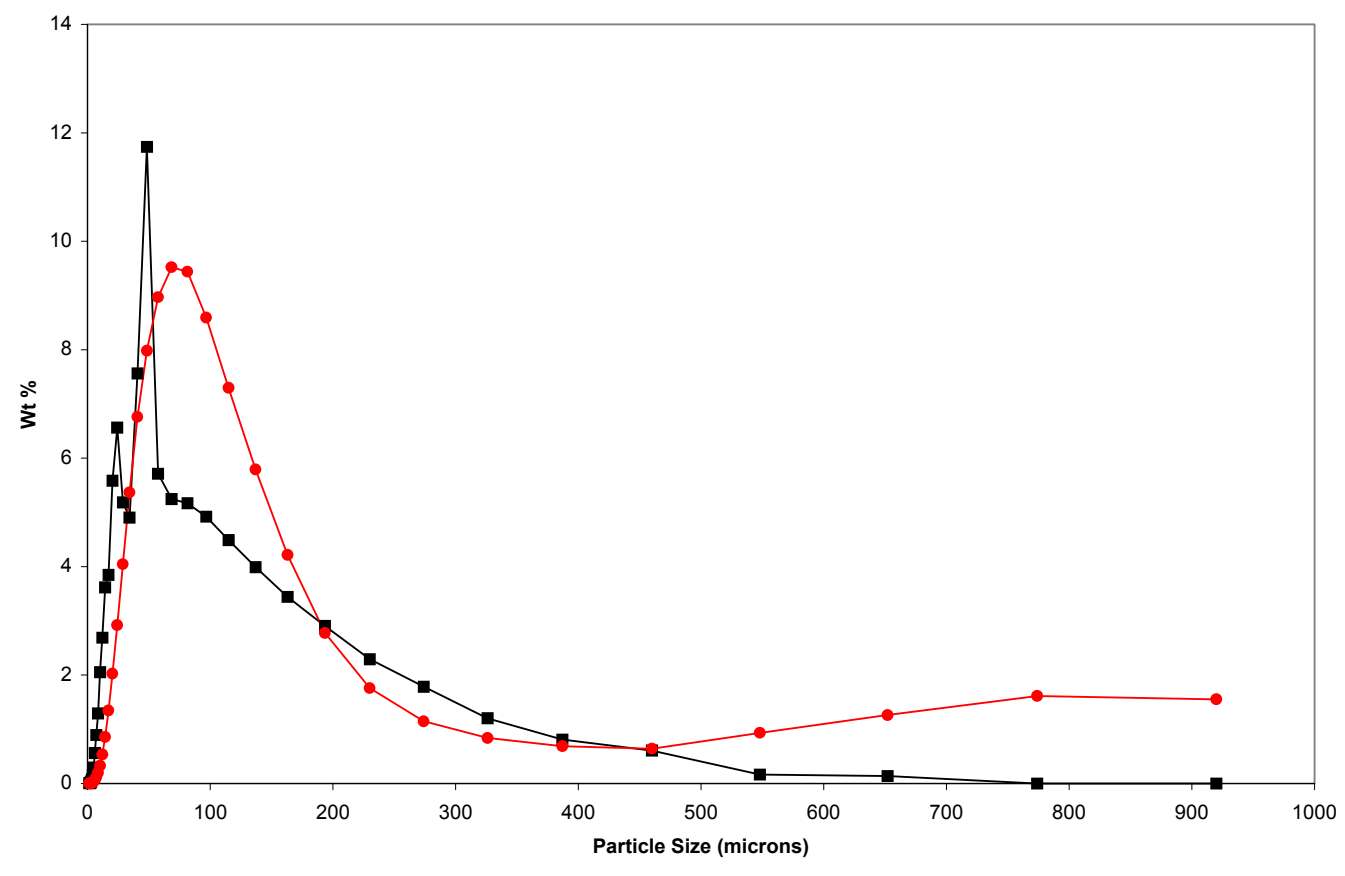

Fig. 28. PSD by weight for selected transfers of waste from tank TH-4. 


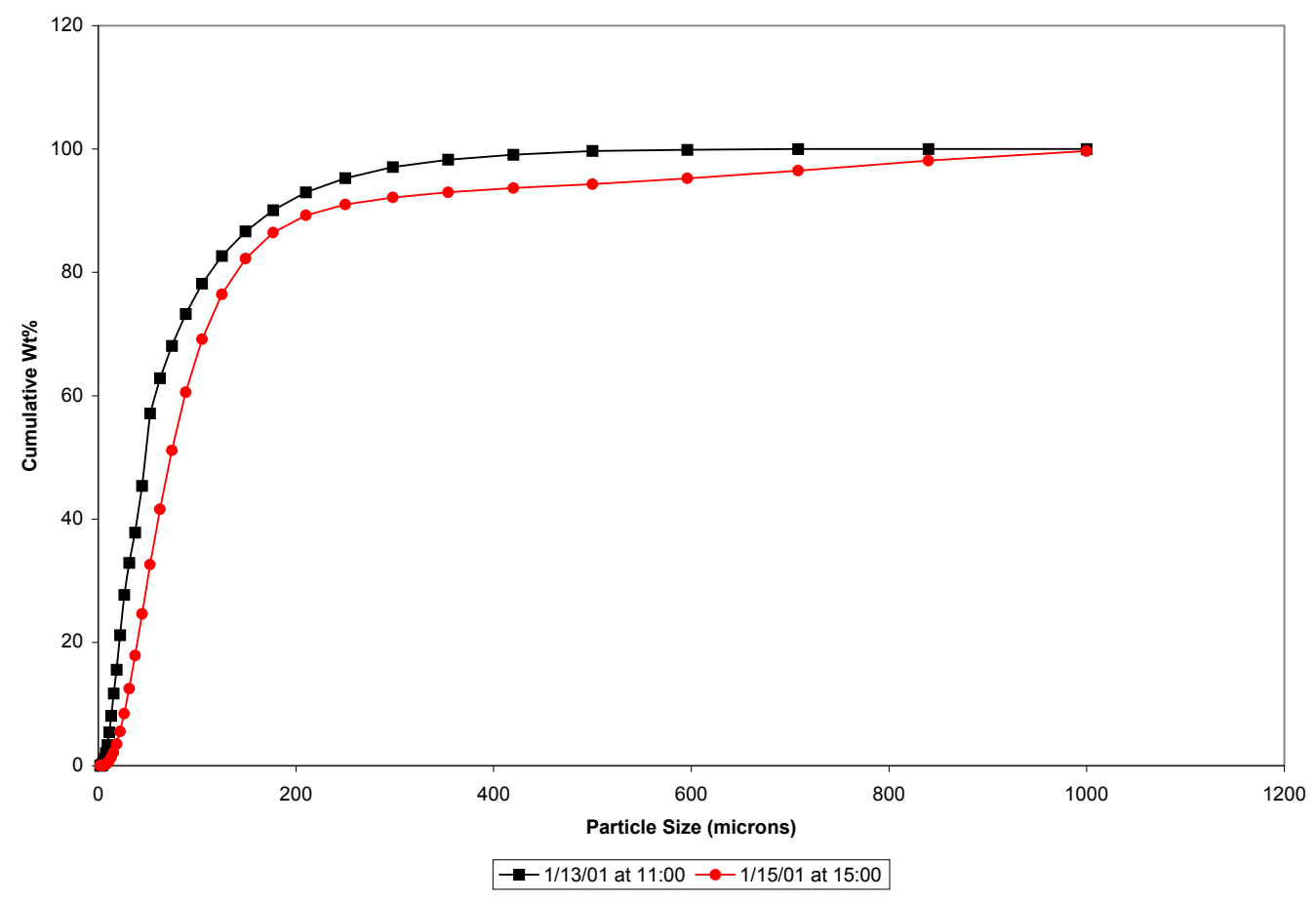

Fig. 29. Cumulative PSD by weight for selected transfers of waste from tank TH-4. 


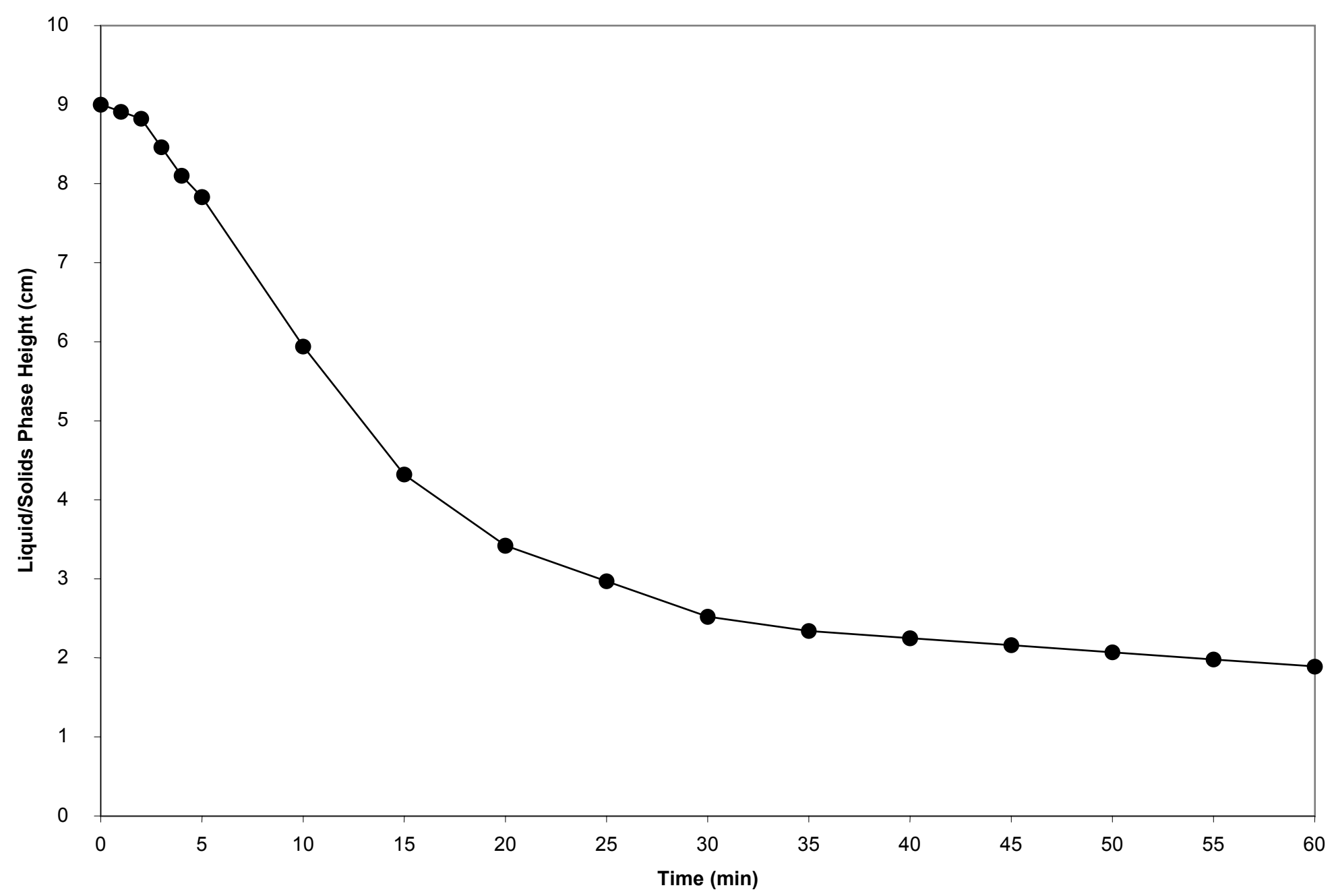

Fig. 30. Settling rate on tank W-23 sludge following retrieval of waste from tank TH-4, ca. January 15, 2001. Starting volume is $50 \mathrm{~mL}$. 


\section{SUMMARY AND LESSONS LEARNED}

\subsection{SUMMARY}

After the sludge had been removed from the first seven Gunite tanks and consolidated into tank W-9, perhaps the greatest challenge remained. The sludge had to be removed from tank W-9 and transferred to the MVST. Pumping the sludge slurry to the MVST without plugging the ICPL became the challenging task. The ICPL is more than a mile long, traverses elevation changes of $200 \mathrm{ft}$, and is only 2-in. in diameter. Furthermore, it had not been used to move solids for a period of several years prior to the GAAT Remediation Project. If this line were plugged, it would shut down all radiochemical operations in the main plant area of ORNL.

Transferring the sludge also brought new administrative challenges. There were new nuclear safety requirements because the project was operating a single tank containing the sludge that was previously dispersed among eight tanks. Also, regulators were relatively less interested in the project's ability to move sludge from tank to tank than they were in getting the sludge out of the main plant area and into the MVST.

When all the challenges are taken into consideration, the performance and effectiveness of the WCS was impressive. In a 16-month period, the WCS succeeded in moving 654,000 gal of waste (total of both solids and liquid) from the STF to the MVSTs ( 483,000 gal), W-9 to the BVESTs ( 147,000 gal), and from TH-4 to the BVESTs ( 24,000 gal). In addition to the solid and liquid wastes a total of $\sim 420,500$ gal of fresh water (used in sluicing operations) was transferred. Including the sluice water, the total volume of material transferred was $\sim 1,074,500$ gal. The solids portion of that total is $\sim 94,000$ gal. The transfers were accomplished without significant modification, maintenance, or change in operation of any of the WCS system components, with three exceptions. These exceptions were (1) the perceived reliability of the slurry density measurements from instruments in the SMTL (and the utility of temperature, viscosity, etc.); (2) the bypassing of the particle size classifiers in the PCS, in part because the LasenTec data on particle size indicated they were not really needed; and (3) the initial difficulties in getting the Discflo pump online. Table 5 displays the approximate run times for the various equipment systems.

Table 5. Run time (in hours) of WCS component systems

\begin{tabular}{lcc}
\hline WCS component system & $\begin{array}{c}\text { Deployment } \\
\text { location }\end{array}$ & $\begin{array}{c}\text { Total operation } \\
\text { (h, approximate) }\end{array}$ \\
\hline PulsAir & W-9 & $2,362.0$ \\
Flygt mixer & W-9, W-5 & $322.5^{1}$ \\
Russian PMP & TH-4 & 30.0 \\
Discflo pump (WTP) & W-9 & 180.0 \\
PCS & STF & 180.0 \\
SMTL & STF & 210.0 \\
WaRTS & W-9 & 85.5 \\
\hline
\end{tabular}

${ }^{l}$ Does not include operating time while mixer was installed in $\mathrm{W}-5$.

In summary, the PAM system proved to be the most reliable of all the WCS component systems. It operated in tank W-9 from January 1999 to March 2000 when it was removed from the tank to make way for WaRTS and the in-tank robotic waste retrieval systems. The system was effective in suspending 
sludge from an inverted cone estimated at several feet in diameter around each accumulator plate. It was not effective in preventing larger particles from settling to the bottom of the tank away from the influence of the accumulator plates.

A Flygt mixer was added to the original WCS configuration as a matter of necessity and convenience. It was evident to project managers that something more than the PAM system would be needed to agitate the heavy waste in tank W-9 during the final cleanout. One of the Flygt mixers was moved from W-5 to fulfill this need. Because the Flygt mixer could be adjusted directionally, some success was achieved at "aiming" the flow of the mixer at the mounds of sludge that were not being affected by the PAM. This additional capability was a significant contribution to the final cleanout effort.

The Russian PMP was an adequate system to mobilize waste in the relatively small TH-4 tank. However, the percentage of total waste (sludge and supernate) removed from this tank was less than any of the other GAAT tanks. It is estimated that $\sim 93.5 \%$ of the waste was removed from TH-4 compared with an average among the other tanks of $\sim 99.4 \%$.

The Discflo pump was a vital component in the overall success of the WCS. Regulators with the State of Tennessee and the EPA had made it clear that the removal of waste from the STF to the MVST was the ultimate goal of the entire project. The Discflo pump functioned almost flawlessly after the initial technical problems were resolved. Problems with the electric power feed caused an early failure of the variable frequency drive unit and was easily corrected. The pump was designed to operate while submerged in water, and when the liquid level in the tank dropped below the motor housing, the part overheated. Using a secondary pump to transfer a stream of supernatant across the housing solved the cooling problem.

The PCS performed well with two of the four key functions it was designed to do. The Isolock samplers and the pressure transmitter were vital in monitoring the transfer of waste from tank W-9 to the MVST or W-23. The classifiers in the PCS were used only once because of (1) difficulties with blinding of the classifiers by the sticky solids and (2) the evidence provided by the LasenTec instrument and sample analysis data that showed that the solid particles were typically $<100 \mu \mathrm{m}$. The PCS was also designed to accept a solids grinder, but it was not needed and was not installed. However, that flexibility did permit installation of the air-operated diaphragm pump used by the WaRTS to transfer waste to the BVEST.

The SMTL proved to be of critical value to the GAAT project in regards to providing the credible data needed to demonstrate that the slurry met the particle size limits of the WAC for the waste transfer line. This capability took on even greater importance when the PCS classifiers were bypassed. However, the inability of the SMTL to provide credible slurry density measurements meant that samples had to be taken during every transfer to verify nuclear safety limits were met and to maintain a material balance.

The WaRTS performed as designed. Its only operational difficulty was that the air sparger in the bottom of the surge tank became plugged. However, this did not have an adverse effect on the tank clean-out process.

The doubly contained waste pipeline (flexible hose inside a PVC pipe), constructed to temporarily connect tank TH-4 to the WCS enclosure, was adequate for the brief clean-out campaign for that tank.

\subsection{LESSONS LEARNED}

Several lessons were learned during the waste transfer phase of the GAAT project. The following three are discussed in this section: (1) maintaining air pressure at all times on the PAM system is required to 
avoid plugging of the accumulator plates; (2) lightning arrestors for the PAM controllers are required; and (3) adequate cold testing of equipment is needed.

\subsubsection{Air Pressure on PAM}

The PAM system was installed in tank W-9 in June 1998, was tested briefly, but was shut-off and not operated again until December 1998. During this period, one of the accumulator plates became plugged with sludge. An unsuccessful attempt was made to clear the sludge with pressurized air. The plug was finally cleared with hydrostatic pressure. Considering the operational reliability of the PAM system, running the system periodically (even if it is not needed) will avoid plugging the accumulator plates.

\subsubsection{Lightning Arrestor on PAM Controller}

The PAM system was operated around the clock in stretches of several days at a time. The most attention the PAM system seemed to need was to reset it following electrical storms. A lightning arrestor might provide additional reliability.

\subsubsection{Adequate Cold Testing of Equipment and Systems}

Additional cold testing and qualification of the Discflo pump should have been conducted to improve the initial success of the system in the GAAT. The Discflo pump was tested by the vendor in the presence of ORNL personnel and shipped to ORNL, where it was subsequently stored for several months prior to deployment. No additional qualification or cold testing at ORNL was performed because of the modifications required at the test facilities to accommodate the pump and because of schedule considerations. On-site qualification consisted of the attachment of power leads to briefly "bump" the motor. Following this brief test, the Discflo pump was installed in tank W-9.

During the initial testing and installation of the Discflo pump in tank W-9, an electrical breaker on the 480-V power supply to the pump tripped open after a few hours of operation and could not be reset. Troubleshooting revealed a problem with the variable frequency drive, which was sent to a local authorized service contractor for diagnosis. The drive had been damaged by extremely low line impedance and a current imbalance across the phases on the ORNL power system. Installation of an isolation transformer and load reactor corrected the situation.

These problems manifested themselves during the U.S. Department of Energy (DOE) readiness assessment for the initiation of waste transfer operations and may have been identified earlier if a complete qualification and cold testing of the equipment had been performed. It is not certain, however, that the problem would have been identified simply through testing. Operation at the different locations of the GAAT and test facility implies that different electrical supply circuits were used. Thus, complete qualification must include analysis or testing of equipment for susceptibility to power supply irregularities. The timing brought the most intense scrutiny experienced during any phase of the GAAT project. At stake was the ability to transfer waste slurry from the STF, which was the fundamental interest of regulators and DOE. The challenge to correct the problems was compounded by the fact the problems surfaced after the Discflo pump had been installed in tank W-9, a highly contaminated environment. The lesson learned from this situation cannot be overstated - all equipment, especially mission-critical, expensive, or long lead-time equipment should be thoroughly cold-tested and qualified prior to deployment in a hostile environment where maintenance and repair become difficult and costly. 


\section{REFERENCES}

Autry, J. W. et al., 1990. Sampling and Analysis of the Inactive Waste Storage Tank Contents at ORNL. ORNL/ER-13. Oak Ridge National Laboratory, Oak Ridge, Tennessee, May.

DOE (U.S. Department of Energy) 2001. Remedial Action Report on the Gunite ${ }^{\mathrm{TM}}$ and Associated Interim Remedial Action Project at the Oak Ridge National Laboratory, Oak Ridge, Tennessee. Draft, Oak Ridge, Tennessee.

Giaquinto, J. M., J. M. Keller, and T. P. Mills. 1997. Miscellaneous Data for the 1996-1997 Sampling and Analysis Campaigns of the MVST, BVEST, and OHF Tank Complexes. ORNL/TM-13455. Oak Ridge National Laboratory, Oak Ridge, Tennessee, July.

Hatchell, B., B. E Lewis, J. D. Randolph, and M. Johnson. 2001. Russian Pulsating Mixer Pump Deployment in the Gunite and Associated Tanks at ORNL. PNNL-SA-34056. Pacific Northwest National Laboratory, Richland, Washington, March.

Holder, L., Jr. 1993. Phase 1-Safety Analysis Report (SAR) Update Program Hazard Screening--South Tank Farm Facility 3507. ORNL/M-2578. Oak Ridge National Laboratory, Oak Ridge, Tennessee, January.

Hylton, T. D. et al., 1998. Comparative Testing of Slurry Monitors. ORNL/TM-13587. Oak Ridge National Laboratory, Oak Ridge, Tennessee, May.

Hylton, T. D., and C. K. Bayne. 1999. Testing of In-Line Slurry Monitors and Pulsair Mixers with Radioactive Slurries. ORNL/TM-1999/111. Oak Ridge National Laboratory, Oak Ridge, Tennessee, July.

Innovative Technology. 2000. Summary Report, Heavy Waste Retrieval System. OST/TMS ID 2194. October.

Lewis, B. E et al., 2000. Basis for Selection of a Residual Waste Retrieval System for Gunite and Associated Tank W-9 at the Oak Ridge National Laboratory. ORNL/TM-2000/251. Oak Ridge National Laboratory, Oak Ridge, Tennessee, September.

Lewis, B. E, and J. D. Randolph. 2001. Cold Testing of a Russian Pulsating Mixer Pump at the Oak Ridge National Laboratory, Oak Ridge, Tennessee, ORNL/TM-2001/141. Oak Ridge National Laboratory, Oak Ridge, Tennessee, December.

ORNL (Oak Ridge National Laboratory). 1998. Cold Test Summary Report for Bulk Sludge Retrieval Systems for the Gunite and Associated Tanks. Letter Report, Oak Ridge, Tennessee, July 31.

Randolph, J. D. et al., 2000. Fabrication of a Sludge-Conditioning System for Processing Legacy Wastes from the Gunite and Associated Tanks. ORNL/TM-2000/222. Oak Ridge National Laboratory, Oak Ridge, Tennessee, August.

Rule, V. A., B. A. Burks, S. D. Van Hoesen. 1998. North Tank Farm Data Report for the Gunite and Associated Tanks at Oak Ridge National Laboratory Oak Ridge, Tennessee. ORNL/TM-13630. Oak Ridge National Laboratory, Oak Ridge, Tennessee, May. 



\section{INTERNAL DISTRIBUTION}

1. J. N. Herndon

2. R. T. Jubin

3. S. M. Killough

4. B. E Lewis

5. P. D. Lloyd

6. J. D. Randolph
7. B. B. Spencer

8. H. Toy

9. S. D. Van Hoesen

10. ORNL Central Research Library

11. ORNL Laboratory Records - OSTI

12. ORNL Laboratory Records- $-\mathrm{RC}$

\section{EXTERNAL DISTRIBUTION (Electronic)}

13. J. A. Emison, Informatics, Inc.

14. P. W. Gibbons, Numatech Hanford Co.

15. M. A. Johnson, Tetra Tech, Inc.

16. J. R. Noble-Dial, U.S. DOE Oak Ridge Operations

\section{EXTERNAL DISTRIBUTION (Paper)}

17. K. D. Gerdes, DOE Office of Science and Technology, 19901 Germantown Road, 1154 Cloverleaf Building, Germantown, MD, 20874-1290

18. T. P. Pietrock, U.S. Department of Energy, Richland Operations Office, PO Box 550, MS: K8-50, Richland, WA 99352

19 B. J. Williams, Pacific Northwest National Laboratory, PO Box 999, MSIN K9-69, Richland, WA 99352 